A. YAMADA

nODAI MATH. J.

3 (1980), 114-143

\title{
PRECISE VARIATIONAL FORMULAS FOR ABELIAN DIFFERENTIALS
}

\author{
BY AKIRA YAMADA
}

In the present paper, we shall study two basic types of degenerations of compact Riemann surfaces considered by Schiffer-Spencer [10] and Fay [3]. According to the simple formalism of the degeneration considered here, the precice variational formulas without error terms will be obtained for $\omega(x, y)$ the fundamental normalized Abelian differentials of the second kind (Theorems 4 and 6), from which one may deduce similar formulas for any Abelian differentials and period matrices in the usual way. It turns out, however, that all the variational formulas found in the book by Fay [3] disagree with ours and it seems to us that they are incorrect, which is, to some extent, seen from the examples in the last section of this paper. In our formulas the coefficients $\beta_{j k}$ of an expansion of $\omega(x, y)$ plays an important role. In this connection a variant of Golusin's inequality will be obtained for $\beta_{j k}$ 's (Theorem 5) which can be viewed as the generalized Faber coefficients. Our method is completely elementary (c.f. Fay [3]) and yields some extension of the results in [3] and [6].

\section{Pinching along a cycle homologous to zero and preliminary estimates.}

On any Riemann surface, it is well-known that the following orthogonal decomposition holds [1]:

$$
\Gamma=\Gamma_{h} \oplus \Gamma_{e o} \oplus \Gamma_{e o}^{*}
$$

where $\Gamma$ is the Hilbert space of square integrable differential forms, $\Gamma_{h}$ its subspace of harmonic differentials, $\Gamma_{e o}$ the closure of the subspace of smooth differentials with compact supports, $I_{e o}^{*}$ the ${ }^{*}$-conjugate of $\Gamma_{e o}$. The above decomposition easily gives a lemma concerning the "distance" between the functions each defined on one of the boundary components of an annulus.

LEMMA. 1. Let $D$ be an annulus $r<|z|<R$ and assume that $\phi(z)$ (resp. $\phi(z))$ is holomorphic on $|z|=r$ (resp. $|z|=R$ ), where they have the Laurent expansions

$$
\phi(z)=\sum_{n=-\infty}^{\infty} a_{n} z^{n}, \quad \phi(z)=\sum_{n=-\infty}^{\infty} b_{n} z^{n} .
$$

Received April 3, 1979 
Then the Dirnclet norm $\|d f-i * d f\|_{D}$ attains its minimum among the (non-vord) family

$$
\mathscr{I}=\left\{f \in C(\bar{D}) \cap C^{1}(D) ;\|d f\|_{D}<\infty, f=\phi \text { on }|z|=r, f=\psi \text { on }|z|=R\right\}
$$

if and only if $f$ is harmonic. Moreover, the minımum is given by:

$$
\min _{f \in \mathscr{F}} \frac{1}{2 \pi}\left\|d f_{-i} * d f\right\|^{2}=\sum_{n=-\infty}^{\infty} \frac{n\left|b_{-n}-a_{-n}\right|^{2}}{R^{2 n}-r^{2 n}}+\frac{\left|b_{0}-a_{0}\right|^{2}}{2 \ln R / r}
$$

(The notation $\Sigma^{\prime}$ indicates that in the summation $n \neq 0$.)

Proof. First note that (c.f. Weyl [11] p. 105)

$$
d u-d v \in \Gamma_{e o} \quad \text { for any } u, v \in \mathscr{F} .
$$

The sketch of the proof of (3) goes as follows:

Choose a $\xi \in C^{\infty}(\boldsymbol{R})$ such that

$$
\xi(x)=\left\{\begin{array}{ll}
1, & x \geqq 2 \\
0 & x \leqq 1
\end{array} \quad 0 \leqq \xi(x) \leqq 1\right.
$$

and set up the following function for $\varepsilon>0$ :

$$
\xi_{s}(z)=\xi\left(\frac{(R-|z|)(|z|-r)}{\varepsilon}\right) \in C_{0}^{\infty}(D) .
$$

In order to conclude that

$$
\Gamma_{e o} \ni d\left[\xi_{\varepsilon} \cdot(u-v)\right] \rightarrow d(u-v) \quad(\varepsilon \rightarrow 0),
$$

it is only necessary to use the inequality

$$
\int_{0}^{2 \pi}\left|w\left(\rho e^{i \theta}\right)\right|^{2} d \theta \leqq\left\{\begin{array}{l}
\ln \rho / r \cdot\|d w\|_{r<|z|<\rho}^{2} \\
\ln R / \rho \cdot\|d w\|_{\rho<|z|<R}^{2}
\end{array} \quad(r<\rho<R)\right.
$$

with $w=u-v(u, v \in \mathscr{F})$, evaluating the norm of $w d \xi_{\varepsilon}$.

In view of (3) and the decomposition (1), the first assertion stated in Lemma 1 holds at once. It remains to compute the minimum. An easy calculation shows that the extremal harmonic function $h(z) \in \mathscr{F}$ is given explicitly by:

$$
\begin{aligned}
h(z)= & \sum_{n=-\infty}^{\infty} \frac{R^{2 n} b_{n}-r^{2 n} a_{n}}{R^{2 n}-r^{2 n}} z^{n}+\sum_{n=-\infty}^{\infty} \frac{b_{-n}-a_{-n}}{R^{2 n}-r^{2 n}} \bar{z}^{n} \\
& +\frac{b_{0}-a_{0}}{\ln R / r} \ln |z|+\frac{a_{0} \ln R-b_{0} \ln r}{\ln R / r} .
\end{aligned}
$$

By the identity $d h-i^{*} d h=2 h_{\bar{z}} d \bar{z}$, (2) is immediately obtained and the proof is completed.

Let $S_{1}$ and $S_{2}$ be two compact Riemann surfaces of genus $g_{1}, g_{2}$ each with a point $p_{1}, p_{2}$ fixed and let $z_{1}: U_{1} \rightarrow \Delta=\{z \in C ;|z|<1\}$ and $z_{2}: U_{2} \rightarrow \Delta$ be coor- 
dinates in neighborhoods $U_{1}, U_{2}$ of these points with $z_{\jmath}\left(p_{j}\right)=0(\jmath=1,2)$. Set

$$
\rho U_{\jmath}=\left\{p \in U_{\jmath} ;\left|z_{j}(p)\right|<\rho\right\}, \quad \rho C_{\jmath}=\left\{p \in U_{\jmath} ;\left|z_{j}(p)\right|=\rho\right\} \quad(\jmath=1,2)
$$

with $0<\rho<1$. A family of compact Riemann surfaces $\left\{S_{\varepsilon} ; \varepsilon \in C, 0<|\varepsilon|<1\right\}$ formed from $S_{1}$ and $S_{2}$ is constructed by defining

$$
S_{\varepsilon}=\left(S_{1} \backslash|\varepsilon| U_{1}\right) \cup\left(S_{2} \backslash|\varepsilon| U_{2}\right)
$$

where $x \in U_{1} \backslash|\varepsilon| U_{1}$ is identified with $y \in U_{2} \backslash|\varepsilon| U_{2}$ by the equation

$$
z_{1}(x) z_{2}(x)=\varepsilon \text {. }
$$

The coordinates $z_{1}$ and $z_{2}$ are called the pinching coordinates for $S_{1}$ and $S_{2}$ at $p_{1}$ and $p_{2}$ respectively. Clearly, $S_{\varepsilon}$ is a compact Riemann surface of genus $g=g_{1}+g_{2}$. Both the pinching coordinates map conformally the "pinched region" $S_{\varepsilon} \backslash\left(\left(S_{1} \backslash U_{1}\right) \cup\left(S_{2} \backslash U_{2}\right)\right)$, denoted by $P_{\varepsilon}$, onto the annulus $|\varepsilon|<|z|<1$, so that $S_{\varepsilon}$ may be regarded as the union of $S_{1} \backslash U_{1}, S_{2} \backslash U_{2}$ and $|\varepsilon|<|z|<1$ under appropriate identification.

From Lemma 1, we obtain the following theorem which is the basis for the derivation of the variational formulas in this paper.

THEOREM 1. Let $\Omega$, be a meromorphic differential on $S_{\text {j }}$ which is holomorphic on $U$, except for a possible simple pole at $p$, with residue $(-1)^{\jmath} \alpha(j=1,2)$. Let $\phi_{j}(x)=\int_{p_{\jmath}}^{x}\left(\Omega_{\jmath}-\frac{(-1)^{\jmath} \alpha}{z_{\jmath}} d z_{\jmath}\right)$ in $U_{\jmath}$ and have a Taylor expansion in terms of the coordinates $z$ given by

$$
\phi_{j}(z)=\sum_{n=1}^{\infty} \alpha_{n}^{(j)} z^{n}, \quad|z|<1 \quad(\jmath=1,2),
$$

Then there exists a meromorphic differential $\Omega_{\varepsilon}$ on $S_{\varepsilon}$ which is holomorphic on $P_{\varepsilon}$ with the same singularities as $\Omega_{\jmath}$ on $S_{\jmath} \backslash U_{\jmath}(\jmath=1,2)$, satisfying, for any $\rho \in\left(|\varepsilon|^{1 / 2}, 1\right)$,

$$
\sum_{j=1}^{2}\left\|\Omega_{\varepsilon}-\Omega_{j}\right\|_{S_{j} \uparrow U} \leqq \pi \sum_{n=1}^{\infty} n\left(\left|\alpha_{n}^{(1)}\right|^{2}+\left|\alpha_{n}^{(2)}\right|^{2}\right) \cdot \frac{|\rho \varepsilon|^{2 n}}{\rho^{4 n}-|\varepsilon|^{2 n}} .
$$

Proof. Let $h_{\varepsilon}$ be the harmonic function on an annulus $|\varepsilon| / \rho \leqq|z| \leqq \rho$ such that

$$
h_{\varepsilon}(z)= \begin{cases}\phi_{1}(z)=\sum_{n=1}^{\infty} \alpha_{n}^{(1)} z^{n} & \text { on } \quad|z|=\rho, \\ \phi_{2}(\varepsilon / z)=\sum_{n=1}^{\infty} \alpha_{n}^{(2)} \varepsilon^{n} z^{-n} & \text { on } \quad|z|=|\varepsilon| / \rho .\end{cases}
$$

Then, by Lemma 1 , it is seen that 
(7)

$$
\begin{aligned}
\frac{1}{2 \pi}\left\|d h_{\varepsilon}-i^{*} d h_{\varepsilon}\right\|_{|\varepsilon| / \rho<|z|<\rho}^{2} & =\sum_{n=1}^{\infty} \frac{n\left|\alpha_{n}^{(2)} \varepsilon^{n}\right|^{2}}{\rho^{2 n}-|\varepsilon|^{2 n} / \rho^{2 n}}+\sum_{n=-1}^{\infty} \frac{n\left|\alpha_{-n}^{(1)}\right|^{2}}{\rho^{2 n}-|\varepsilon|^{2 n} / \rho^{2 n}}- \\
& =\sum_{n=1}^{\infty} n\left(\left|\alpha_{n}^{(1)}\right|^{2}+\left|\alpha_{n}^{(2)}\right|^{2}\right) \frac{|\rho \varepsilon|^{2 n}}{\rho^{4 n}-|\varepsilon|^{2 n}} .
\end{aligned}
$$

By passing to the usual smoothing process, it is easy to find an $h_{\varepsilon}^{(n)}(n=1,2, \cdots)$ with the same boundary value as $h_{\varepsilon}$ satisfying

(i) $\left\|d h_{\varepsilon}^{(n)}-i^{*} d h_{s}^{(n)}\right\|_{|\varepsilon| / \rho<|z|<\rho}^{2}<\left\|d h_{\varepsilon}-i^{*} d h_{\varepsilon}\right\|_{|\varepsilon| / \rho<|z|<\rho}^{2}+\frac{1}{n}$,

(ii) If we define $\Phi_{\varepsilon}^{(n)}$ on $S_{\varepsilon}$ by

$$
\Phi_{\varepsilon}^{(n)}(z)= \begin{cases}\Omega_{1}(z), \quad z \in S_{1} \backslash \rho U_{1} \\ d\left(h_{\varepsilon}^{(n)}(z)\right)-\frac{d z}{z}, & z \in\{z ;|\varepsilon| / \rho \leqq|z| \leqq \rho\} \\ \Omega_{2}(z), & z \in S_{2} \backslash \rho U_{2},\end{cases}
$$

then $\Phi_{\varepsilon}^{(n)} \in \Gamma_{c}^{1}\left(S_{\varepsilon}\right)$, the space of closed $C^{1}$-differentials $\in \Gamma\left(S_{\varepsilon}\right)$.

Here the coordinate $z_{1}$ is used to identify the pinched region with $|\varepsilon| / \rho<|z|<\rho$. Note that $\left(^{*}\right)$ is well-defined, because of (5) and the restriction imposed on the residues of $\Omega_{1}$ and $\Omega_{2}$ at $p_{1}$ and $p_{2}$. Clearly,

$$
\Phi_{\varepsilon}^{(n)}-i^{*} \Phi_{\varepsilon}^{(n)}= \begin{cases}0, & z \in\left(S_{1} \backslash \rho U_{1}\right) \cup\left(S_{2} \backslash \rho U_{2}\right) \\ d h_{s}^{(n)}-i^{*} d h_{\varepsilon}^{(n)}, & z \in\{|\varepsilon| / \rho<|z|<\rho\} .\end{cases}
$$

From the decomposition (1), it follows that

$$
\Phi_{\varepsilon}^{(n)}-i^{*} \Phi_{\varepsilon}^{(n)}=\omega_{h}^{(n)}+\omega_{e o}^{(n)}+\omega_{e o}^{*(n)}
$$

where $\omega_{h}^{(n)} \in \Gamma_{h}, \omega_{e o}^{(n)} \in \Gamma_{e o}$, and $\omega_{e o}^{*(n)} \in \Gamma_{e o}^{*}$. Let $\tau_{\varepsilon}^{(n)}=\Phi_{\varepsilon}^{(n)}-\omega_{e o}^{(n)}$, then $\tau_{\varepsilon}^{(n)}$ is closed and co-closed. It is also square integrable off the poles of $\Omega_{1}$ and $\Omega_{2}$, so that it is harmonic there by Weyl's lemma. Noting the fact that any harmonic differential with isolated singularities is never square integrable, we see that $\tau_{\varepsilon}^{(n)}$ is harmonic on $S_{\varepsilon}$ except for the same singularities as $\Omega_{1}$ and $\Omega_{2}$ off the pinched region. Now let us define $\Omega_{s}^{(n)}$ by

$$
\begin{aligned}
\Omega_{\varepsilon}^{(n)} & =\frac{1}{2}\left(\tau_{\varepsilon}^{(n)}+i^{*} \tau_{\varepsilon}^{(n)}\right) \\
& =\frac{1}{2}\left(\Phi_{\varepsilon}^{(n)}+i^{*} \Phi_{\varepsilon}^{(n)}-\omega_{e o}^{(n)}-i^{*} \omega_{e o}^{(n)}\right) .
\end{aligned}
$$

Then $\Omega_{\varepsilon}^{(n)}$ is meromorphic on $S_{\varepsilon}$ with the same singularities as $\tau_{\varepsilon}^{(n)}$ and the following estimate holds :

$$
\sum_{j=1}^{2}\left\|\Omega_{\varepsilon}^{(n)}-\Omega_{j}\right\|_{S_{j} \backslash \rho U}^{2}=\frac{1}{4} \sum_{j=1}^{2}\left\|\omega_{e o}^{(n)}+i * \omega_{e o}^{(n)}\right\|_{S_{j} \backslash \rho U}^{2}
$$




$$
\begin{aligned}
& \leqq \frac{1}{4}\left\|\omega_{e o}^{(n)}+i^{*} \omega_{e o}^{(n)}\right\|_{S_{\varepsilon}}^{2}=\frac{1}{2}\left\|\omega_{e o}^{(n)}\right\|_{S_{\varepsilon}}^{2} \\
& \leqq \frac{1}{2}\left\|\Phi_{\varepsilon}^{(n)}-i^{*} \Phi_{\varepsilon}^{(n)}\right\|_{S_{\varepsilon}}^{2} .
\end{aligned}
$$

Here we used the orthogonal decomposition (1). Thus, from (8),

$$
\sum_{j=1}^{2}\left\|\Omega_{\varepsilon}^{(n)}-\Omega_{j}\right\|_{S_{j} \rho \rho U j}^{2} \leqq \frac{1}{2}\left\|d h_{\varepsilon}-i^{*} d h_{\varepsilon}\right\|_{|\varepsilon| / \rho<|z|<\rho}^{2}+\frac{1}{2 n}
$$

for $n=1,2, \cdots$. By a normal family argument, a properly chosen subsequence of $\left\{\Omega_{\varepsilon}^{(n)}\right\}_{n=1}^{\infty}$ converges to a meromorphic differential $\Omega_{\varepsilon}$ on $S_{\varepsilon}$ uniformly off the poles of $\Omega_{1}$ and $\Omega_{2}$. Letting $n \rightarrow \infty$, we conclude that

$$
\sum_{j=1}^{2}\left\|\Omega_{\varepsilon}-\Omega_{j}\right\|_{S_{j} \backslash \rho U}^{2} \leqq \frac{1}{2}\left\|d h_{\varepsilon}-i^{*} d h_{\varepsilon}\right\|_{|\varepsilon| / \rho<|z|<\rho}^{2} .
$$

By combining (7) and (9), the proof is completed.

The above theorem is slightly stronger than what is needed for later applications. Indeed, it is sufficient to obtain the estimate

$$
\sum_{j=1}^{2}\left\|\Omega_{\varepsilon}-\Omega_{j}\right\|_{S_{j} \backslash U_{j}}<A \varepsilon \quad(\varepsilon \rightarrow 0)
$$

with some information about the bound for the constant $A$. If (10) is rewritten in the form

$$
\sum_{\jmath=1}^{2}\left\|\Omega_{\varepsilon}-\Omega_{\jmath}\right\|_{S_{j} \backslash U_{\jmath}}=O(\varepsilon),
$$

the constant $A$ will be called an "implied constant" of the estimate (10)'. After obtaining variational formulas, we will see that the estimate $O(\varepsilon)$ in $(10)^{\prime}$ cannot be replaced by $o(\varepsilon)$ in general.

\section{Derivation of variational formulas.}

Let us fix, once and for all, a canonical homology basis $\left(A^{(\jmath)}, B^{(\jmath)}\right)$ for $S$, where $A^{(1)}=\left(A_{1}, \cdots, A_{g_{1}}\right), \quad B^{(1)}=\left(B_{1}, \cdots, B_{g_{1}}\right), \quad A^{(2)}=\left(A_{g_{1}+1}, \cdots, A_{g}\right)$ and $B^{(2)}=\left(B_{g_{1}+1}, \cdots, B_{g}\right)$, and assume that every cycle in $\left(A^{(\jmath)}, B^{(\jmath)}\right)$ is contained in $S_{j} \backslash U_{j}(j=1,2)$ without loss of generality. To choose some canonical homology basis for $S_{\varepsilon}$, let $A_{1}(\varepsilon), B_{1}(\varepsilon), \cdots, A_{g}(\varepsilon), B_{g}(\varepsilon)$ simply be a canonical basis $A_{1}, B_{1}, \cdots, A_{g}, B_{g}$ for $S_{1}$ and $S_{2}$. Let $v_{\jmath, \varepsilon}(\jmath=1,2, \cdots, g)$ be the normalized differential of the first kind on $S_{\varepsilon}$ such that

$$
\int_{A_{k}(\varepsilon)} v_{\jmath, \varepsilon}=2 \pi i \delta_{j k} \quad(\jmath, k=1, \cdots, g)
$$

where $\delta_{j k}$ is the Kronecker $\delta$. This normalization is used throughout the present paper. 
Let $O$ be a relatively compact region of a Riemann surface, and assume that $u$ is a nowhere-vanishing holomorphic differential on the closure $\bar{O}$. Then a differential $v$ defined in $O$ is said to be bounded if so is the function $v / u$. This definition is clearly independent of the choice of $u$.

The uniform boundedness of $v_{j, \varepsilon}(\jmath=1, \cdots, g)$ with respect to $\varepsilon$ will now be considered, which is crucial for the later development.

LEMMA 2. Let $z \in\left(S_{1} \backslash \rho U_{1}\right) \cup\left(S_{2} \backslash \rho U_{2}\right)$ with $0<\rho<1$. Then, for $\jmath=1, \cdots, g$,

$$
v_{\jmath, \varepsilon}(z)=O(1) \quad(\varepsilon \rightarrow 0)
$$

uniformly. (Here and hereafter estrmates like $f_{\varepsilon}(z)=O\left(\left(\varepsilon-\varepsilon_{0}\right)^{n}\right)\left(\varepsilon \rightarrow \varepsilon_{0}\right)$ are sand to be uniform if "implied constants" can be chosen independently of the variable z.)

Proof. Choose the pairs of differentials $\Omega_{1}^{(j)}$ and $\Omega_{2}^{(j)}$ on $S_{1}$ and $S_{2}$ respectively as follows :

$$
\left(\Omega_{1}^{(j)}, \Omega_{2}^{(j)}\right)=\left\{\begin{array}{lll}
\left(v_{\jmath}, 0\right) & \text { if } & 1 \leqq \jmath \leqq g_{1}, \\
\left(0, v_{j}\right) & \text { if } & g_{1}<j \leqq g,
\end{array}\right.
$$

where $v$, for $\jmath \leqq g_{1}$ (resp. $j>g_{1}$ ) are a normalized basis for the holomorphic differentials on $S_{1}$ (resp. $S_{2}$ ). Then, by applying Theorem 1 , there exists a differential $\Omega_{\jmath, \varepsilon}$ holomorphic on $S_{\varepsilon}$ such that, for $\varepsilon \rightarrow 0$,

$$
\begin{array}{ll}
\left\|\Omega_{j, \varepsilon}-v_{j}\right\|_{S_{1} \backslash \rho U_{1}}+\left\|\Omega_{\jmath, \varepsilon}\right\|_{S_{2} \backslash \rho U_{2}}=O(\varepsilon), & 1 \leqq \jmath \leqq g_{1}, \\
\left\|\Omega_{\jmath, \varepsilon}\right\|_{S_{1} \backslash \rho U_{1}}+\left\|\Omega_{\jmath, \varepsilon}-v_{j}\right\|_{S_{2} \backslash \rho U_{2}}=O(\varepsilon), & g_{1}<j \leqq g .
\end{array}
$$

Since this holds for any $\rho \in(0,1)$, it follows immediately that

$$
\Omega_{\jmath, \varepsilon}(z)=O(1) \quad(\varepsilon \rightarrow 0)
$$

uniformly for $z \in\left(S_{1} \backslash \rho U_{1}\right) \cup\left(S_{2} \backslash \rho U_{2}\right)$. Let $M_{\varepsilon}$ be the period matrix of $\Omega_{1, \varepsilon}, \cdots$, $\Omega_{g, \varepsilon}$ with respect to the cycles $A_{1}(\varepsilon), \cdots, A_{g}(\varepsilon)$. Then one verifies that

$$
M_{\varepsilon}=\left(\frac{1}{2 \pi i} \int_{A_{j}(\varepsilon)} \Omega_{k, \varepsilon}\right)_{J, k=1}^{g}=I_{g}+O(\varepsilon), \quad(\varepsilon \rightarrow 0)
$$

where $I_{g}$ is the $g \times g$ identity matrix, since the period along a fixed cycle is a bounded linear functional on $\Gamma_{c}$, the space of closed square integrable differentials (c.f. Ahlfors-Sario [1], p. 284). Therefore the inverse matrix $M_{\varepsilon}^{-1}$ exists for $\varepsilon$ sufficiently small and is of the form

Consequently,

$$
M_{\varepsilon}^{-1}=I_{g}+O(\varepsilon) \text {. }
$$

$$
\left(v_{j, \varepsilon}\right)_{j=1}^{g}=M_{\varepsilon}^{-1}\left(\Omega_{\jmath, \varepsilon}\right)_{j=1}^{g}=\left(\Omega_{\jmath, \varepsilon}\right)_{j=1}^{g}+O(\varepsilon)\left(\Omega_{\jmath, \varepsilon}\right)_{\jmath=1}^{g}=O(1) \quad(\varepsilon \rightarrow 0) .
$$

This completes the proof. 
Let $\omega_{1}(x, y)$ (resp. $\left.\omega_{2}(x, y), \omega_{\varepsilon}(x, y)\right)$ be the fundamental normalized differential of the second kind on $S_{1}$ (resp. $S_{2}, S_{\varepsilon}$ ), that is, the bilinear meromorphic differential with vanishing $A_{j}$-periods which is holomorphic everywhere except for a double pole along $x=y$, where, in terms of a coordinate, it has an expansion given by

$$
\frac{d x d y}{(x-y)^{2}}+\text { regular terms. }
$$

For $x \in S_{1} \backslash U_{1}$ (resp. $x \in S_{2} \backslash U_{2}, x \in S_{\varepsilon} \backslash P_{\varepsilon}$ ), let the following expansions, in terms of the pinching coordinates, hold in $U_{1}$ (resp. $U_{2}, P_{\varepsilon}$ ):

$$
\begin{array}{ll}
\int_{0}^{z} \omega_{j}(\cdot, x)=\sum_{n=1}^{\infty} a_{n}^{(j)}(x) z_{\jmath}^{n}, & \left|z_{j}\right|<1 \quad(\jmath=1,2) \\
\int^{z_{1}} \omega_{\varepsilon}(\cdot, x)=\sum_{n=-\infty}^{\infty} a_{n, \varepsilon}(x) z_{1}^{n}, & |\varepsilon|<\left|z_{1}\right|<1 .
\end{array}
$$

Here the constant term $a_{o, \varepsilon}(x)$ needs not to be determined. The coefficients $a_{n}^{(j)}(x)$ are easily seen to be extended so that these become normalized differentials of the second kind on $S$, holomorphic everywhere except for a pole of order $n+1$ at $p$, where, in terms of the pinching coordinates,

$$
\begin{aligned}
& a_{1}^{(j)}(z)=\omega_{j}\left(z, p_{j}\right) \\
& a_{n}^{(j)}\left(z_{j}\right)=d z_{j} / z_{j}^{n+1}+\text { regular terms } \quad(\jmath=1,2 ; n=1,2, \cdots) .
\end{aligned}
$$

LEMMA 3. The following uniform estimates hold with $0<\rho<1$ :

$$
\omega_{\varepsilon}(x, y)=\left\{\begin{array}{ll}
\omega_{j}(x, y)+O(\varepsilon), & x, y \in S_{\jmath} \backslash \rho U_{\jmath} \\
O(\varepsilon) \quad, & x \in S_{j} \backslash \rho U_{\jmath}, y \in S_{j^{\prime}} \backslash \rho U_{j^{\prime}}
\end{array} \quad(j=1,2)\right.
$$

Here and hereafter we use the convention that

$$
j^{\prime}= \begin{cases}2, & \jmath=1 \\ 1, & \jmath=2 .\end{cases}
$$

Proof. Set $\Omega_{1}=\omega_{1}(\cdot, x), \Omega_{2}=0$ and apply Theorem 1, assuming that $x \in S_{1} \backslash \rho U_{1}$ without loss of generality. Then there exists a differential $\Omega_{\varepsilon}(\cdot ; x)$ meromorphic on $S_{\varepsilon}$ satisfying, for positive $\rho^{\prime}<\rho$,

$$
\begin{gathered}
\left\|\Omega_{\varepsilon}(\cdot ; x)-\omega_{1}(\cdot, x)\right\|_{S_{1} \backslash \rho^{\prime} U_{1}}+\left\|\Omega_{\varepsilon}(\cdot, x)\right\|_{S_{2} \backslash \rho^{\prime} U_{2}} \\
\leqq \pi \sum_{n=1}^{\infty} \frac{n\left|a_{n}^{(1)}(x)\right|^{2}|\rho \cdot \varepsilon|^{2 n}}{\rho^{\prime 4 n}-|\varepsilon|^{2 n}} .
\end{gathered}
$$

By Cauchy's estimate, it follows that

$$
\left|a_{n}^{(1)}(x)\right| \rho^{\prime n} \leqq K=\operatorname{Max}\left\{\left|\int_{0}^{z} \omega_{1}(\cdot, x)\right| ;|z|<\rho^{\prime}, x \in S_{1} \backslash \rho U_{1}\right\}
$$


Thus

$$
\sum_{n=1}^{\infty} \frac{n\left|a_{n}^{(1)}(x)\right|^{2}\left|\rho^{\prime} \varepsilon\right|^{2 n}}{\rho^{\prime 4 n}-|\varepsilon|^{2 n}} \leqq K^{2} \sum_{n=1}^{\infty} \frac{n|\varepsilon|^{2 n}}{\rho^{\prime 4}-|\varepsilon|^{2 n}}=O\left(\varepsilon^{2}\right)
$$

uniformly in $x \in S_{1} \backslash \rho U_{1}$. Analogous to Lemma 2, the following uniform estimates hold:

$$
\begin{aligned}
& \Omega_{\varepsilon}(y ; x)= \begin{cases}\omega_{1}(x, y)+O(\varepsilon) & x, y \in S_{1} \backslash \rho U_{1}, \\
O(\varepsilon) & x \in S_{1} \backslash \rho U_{1}, y \in S_{2} \backslash \rho U_{2},\end{cases} \\
& \int_{A_{j}(\varepsilon)} \Omega_{\varepsilon}(y ; x)=O(\varepsilon) \quad(j=1,2, \cdots g) .
\end{aligned}
$$

In order to conclude the proof, it is sufficient to note that

$$
\omega_{\varepsilon}(x, y)=\Omega_{\varepsilon}(y ; x)-\sum_{j=1}^{g} \frac{1}{2 \pi i}\left(\int_{A_{j}(\varepsilon)} \Omega_{\varepsilon}(\cdot, x)\right) v_{\jmath, \varepsilon}(y)
$$

and that $v_{\jmath, s}(y)=O(1)$ uniformly by Lemma 2 .

For our later development, it will be useful to derive an identity which comes from the method of contour integration. For simplicity, let us define $\omega_{0}(x, y)$ by :

$$
\omega_{0}(x, y)=\left\{\begin{array}{ll}
\omega_{j}(x, y), & x, y \in S_{\jmath} \\
0 \quad, & x \in S_{\jmath}, y \in S_{j^{\prime}}
\end{array} \quad(\jmath=1,2) .\right.
$$

Lemma 4. Let $\varepsilon, \varepsilon_{o} \in \boldsymbol{C}$ and $\rho \in \boldsymbol{R}$ satisfy $\operatorname{Max}\left\{|\varepsilon|^{1 / 2},\left|\varepsilon_{0}\right|^{1 / 2}\right\}<\rho<1$. Then the following identity holds: for $x, y \in\left(S_{1} \backslash \rho U_{1}\right) \cup\left(S_{2} \backslash \rho U_{2}\right)$

$$
\omega_{\varepsilon}(x, y)-\omega_{\varepsilon_{0}}(x, y)=\frac{1}{2 \pi \imath} \int_{\rho C_{1}+\rho C_{2}}\left(\int^{z} \omega_{\varepsilon_{0}}(x, \cdot)\right) \omega_{\varepsilon}(y, z)
$$

Proof. Case 1. $x, y \in S_{\jmath} \backslash \rho U_{\jmath}(\jmath=1,2)$ : Integration along the boundary of $S_{j} \backslash \rho U$, canonically dissected yields

$$
\omega_{\varepsilon}(x, y)-\omega_{\varepsilon_{0}}(x, y)=\frac{1}{2 \pi \imath} \int_{\rho C_{j}}\left(\int^{z}\left(\omega_{\varepsilon_{0}}(x, \cdot)-\omega_{\varepsilon}(x, \cdot)\right)\right) \omega_{\varepsilon}(y, z) .
$$

Here the Riemann bilinear relation and the residue theorem were used. The term

$$
\int_{\rho C_{j}}\left(\int^{z} \omega_{\varepsilon}(x, \cdot)\right) \omega_{\varepsilon}(y, z)
$$

vanishes because the integrand is holomorphic on $S_{\varepsilon} \backslash\left(S_{j} \backslash \rho U_{j}\right)$ where Cauchy's integral theorem can be applied. On the other hand, the same theorem again shows

$$
\frac{1}{2 \pi \imath} \int_{\rho C_{j^{\prime}}}\left(\int^{z} \omega_{\varepsilon_{0}}(x, \cdot)\right) \omega_{\varepsilon}(y, z)=0
$$


since the integrand is holomorphic on $S_{\jmath^{\prime}} \backslash \rho U_{\jmath^{\prime}}$. This completes the proof of Case 1.

Case 2. $x \in S_{j} \backslash \rho U_{\jmath}$ and $y \in S_{j^{\prime}} \backslash \rho U_{\jmath^{\prime}}(\jmath=1,2)$ : Similar reasoning as above gives

$$
\frac{1}{2 \pi \imath} \int_{\rho C_{j}}\left(\int^{z}\left(\omega_{\varepsilon_{0}}(x, \cdot)-\omega_{\varepsilon}(x, \cdot)\right)\right) \omega_{\varepsilon}(y, z)=0 .
$$

The residue theorem implies

$$
\frac{1}{2 \pi i} \int_{\rho C_{j}}\left(\int^{z} \omega_{\varepsilon}(x, \cdot)\right) \omega_{\varepsilon}(y, z)=\omega_{\varepsilon}(x, y) .
$$

Thus

$$
\omega_{\varepsilon}(x, y)=\frac{1}{2 \pi \imath} \int_{\rho C_{j}}\left(\int^{z} \omega_{\varepsilon_{0}}(x, \cdot)\right) \omega_{\varepsilon}(y, z) .
$$

By symmetry and Stokes' theorem, it is seen that

$$
\begin{aligned}
\omega_{\varepsilon_{0}}(x, y) & =\omega_{\varepsilon_{0}}(y, x)=\frac{1}{2 \pi \imath} \int_{\rho C_{j^{\prime}}}\left(\int^{z} \omega_{\varepsilon}(y, \cdot)\right) \omega_{\varepsilon_{0}}(x, z) \\
& =-\frac{1}{2 \pi \imath} \int_{\rho C_{J^{\prime}}}\left(\int^{z} \omega_{\varepsilon_{0}}(x, \cdot)\right) \omega_{\varepsilon}(y, z) .
\end{aligned}
$$

This completes the proof of Case 2, so that Lemma 4 is proved.

We are now in position to obtain the variational formulas of arbitrary order for $\omega_{1}(x, y)$ and $\omega_{2}(x, y)$. To this end, however, it is important first to recognize that $\omega_{\varepsilon}(x, y)$ is holomorphic in $\varepsilon$. Thus the first or second order variational formulas for $\omega_{\varepsilon}(x, y)$ are needed in advance. Let $\omega_{j}(x, y)$ have an expansion near $x=y=p_{\jmath}$, in terms of the pinching coordinate, given by

$$
\omega_{\jmath}(x, y)=\frac{1}{(x-y)^{2}}+\sum_{k, l=0}^{\infty} \beta_{k l}^{(j)} x^{k} y^{l} \quad(\jmath=1,2) .
$$

THEOREM 2. $\omega_{\varepsilon}(x, y)$ has an expansion

$$
\omega_{\varepsilon}(x, y)=\left\{\begin{array}{l}
\omega_{j}(x, y)+\beta_{00}^{\left(j^{\prime}\right)} \varepsilon^{2} \omega_{j}\left(x, p_{j}\right) \omega_{j}\left(y, p_{j}\right)+O\left(\varepsilon^{3}\right), x, y \in S_{\jmath} \backslash \rho U_{\jmath} \\
-\varepsilon \omega_{j}\left(x, p_{j}\right) \omega_{j^{\prime}}\left(y, p_{j^{\prime}}\right)+O\left(\varepsilon^{2}\right), x \in S_{\jmath} \backslash \rho U_{\jmath}, y \in S_{j^{\prime}} \backslash \rho U_{j^{\prime}}
\end{array}\right.
$$

near $\varepsilon=0$ with $0<\rho<1$. Here the estrmates $O\left(\varepsilon^{2}\right)$ and $O\left(\varepsilon^{3}\right)$ are uniform and the differentials $\omega_{\varepsilon}(x, y), \omega_{j}\left(x, p_{j}\right)$ and $\omega_{j}\left(y, p_{j}\right)$ are all evaluated in terms of the pinching coordinates.

Proof. Let us fix $\rho^{\prime}$ and $\rho^{\prime \prime}$ with $|\varepsilon|^{1 / 2}<\rho^{\prime}<\rho<\rho^{\prime \prime}<1$ and assume that $x \in S_{1} \backslash \rho U_{1}$ without loss of generality. From Lemma 4 with $\varepsilon_{0}=0$, (11) and Cauchy's integral theorem, it is seen that, for $y \in S_{2} \backslash \rho U_{2}$, 


$$
\begin{aligned}
\omega_{\varepsilon}(x, y) & =\frac{1}{2 \pi \imath} \int_{\rho^{\prime} C_{1}}\left(\int_{p_{1}}^{z} \omega_{1}(x, \cdot)\right) \omega_{\varepsilon}(y, z) \\
& =\sum_{n=1}^{\infty} a_{n}^{(1)}(x) \frac{1}{2 \pi \imath} \int_{\rho^{\prime} C_{1}} z_{1}^{n} \omega_{\varepsilon}\left(y, z_{1}\right) \\
& =-\sum_{n=1}^{\infty} \varepsilon^{n} a_{n}^{(1)}(x) \frac{1}{2 \pi i} \int_{\rho^{\prime} C_{2}} \omega_{\varepsilon}\left(y, z_{2}\right) / z_{2}^{n} .
\end{aligned}
$$

Thus Lemma 3 combined with the residue theorem and the equation (12) shows

$$
\begin{aligned}
\omega_{\varepsilon}(x, y) & =-\varepsilon a_{1}^{(1)}(x) \frac{1}{2 \pi \imath} \int_{\rho^{\prime} C_{2}} \omega_{\varepsilon}\left(y, z_{2}\right) / z_{2}+O\left(\varepsilon^{2}\right) \\
& =-\varepsilon \omega_{1}\left(x, p_{1}\right) \frac{1}{2 \pi \imath} \int_{\rho^{\prime} C_{2}} \omega_{2}\left(y, z_{2}\right) / z_{2}+O\left(\varepsilon^{2}\right) \\
& =-\varepsilon \omega_{1}\left(x, p_{1}\right) \omega_{2}\left(y, p_{2}\right)+O\left(\varepsilon^{2}\right) .
\end{aligned}
$$

Here, the estimates $O\left(\varepsilon^{2}\right)$ are all uniform for $x \in S_{1} \backslash \rho U_{1}$ and $y \in S_{2} \backslash \rho U_{2}$.

When $y \in S_{1} \backslash \rho U_{1}$, a similar reasoning shows

$$
\begin{aligned}
\omega_{\varepsilon}(x, y) & =\omega_{1}(x, y)+\frac{1}{2 \pi \imath} \int_{\rho^{\prime} C_{1}}\left(\int_{p_{1}}^{z_{1}} \omega_{1}(x, \cdot)\right) \omega_{\varepsilon}(y, z) \\
& =\omega_{1}(x, y)+\sum_{n=1}^{\infty} a_{n}^{(1)}(x) \frac{1}{2 \pi \imath} \int_{\rho^{\prime} C_{1}} z_{1}^{n} \omega_{\varepsilon}\left(y, z_{1}\right) \\
& =\omega_{1}(x, y)-\sum_{n=1}^{\infty} \varepsilon^{n} a_{n}^{(1)}(x) \frac{1}{2 \pi \imath} \int_{\rho^{\prime} C_{2}} \omega_{\varepsilon}\left(y, z_{2}\right) / z_{2}^{n} .
\end{aligned}
$$

By definition $y \in S_{1} \backslash \rho U_{1}$ and $\rho^{\prime \prime} C_{2} \subset S_{2} \backslash \rho U_{2}$, so that the result already obtained above can be applied to give

$$
\begin{aligned}
\omega_{\varepsilon}(x, y) & =\omega_{1}(x, y)-\varepsilon a_{1}^{(1)}(x) \frac{1}{2 \pi \imath} \int_{\rho^{\prime} C_{2}} \omega_{\varepsilon}\left(y, z_{2}\right) / z_{2}+O\left(\varepsilon^{3}\right) \\
& =\omega_{1}(x, y)+\varepsilon^{2} a_{1}^{(1)}(x) \omega_{1}\left(y, p_{1}\right) \frac{1}{2 \pi \imath} \int_{\rho^{\prime \prime} C_{2}} \omega_{2}\left(p_{2}, z_{2}\right)+O\left(\varepsilon^{3}\right) \\
& =\omega_{1}(x, y)+\beta_{00}^{(2)} \varepsilon^{2} \omega_{1}\left(x, p_{1}\right) \omega_{1}\left(y, p_{1}\right)+O\left(\varepsilon^{3}\right) .
\end{aligned}
$$

Again, the estimates $O\left(\varepsilon^{3}\right)$ are all uniform. This concludes the proof.

THEOREM 3. $\omega_{\varepsilon}(x, y)$ has an expansion near $\varepsilon=\varepsilon_{0}(\neq 0)$

$$
\omega_{\varepsilon}(x, y)=\omega_{\varepsilon_{0}}(x, y)-\frac{\varepsilon-\varepsilon_{0}}{\varepsilon_{0}} \sum_{n=-\infty}^{\infty} n^{2} a_{n, \varepsilon_{0}}(x) a_{-n, \varepsilon_{0}}(y)+O\left(\left(\varepsilon-\varepsilon_{0}\right)^{2}\right)
$$

uniformly for $x, y \in\left(S_{1} \backslash \rho U_{1}\right) \cup\left(S_{2} \backslash \rho U_{2}\right)$ with $\left|\varepsilon_{0}\right|^{1 / 2}<\rho<1$.

Proof. Now let us fix a $\rho^{\prime}$ with $\left|\varepsilon_{0}\right|^{1 / 2}<\rho^{\prime}<\rho$. From Lemma 4 it is seen that 


$$
\begin{aligned}
\omega_{\varepsilon}(x, y)-\omega_{\varepsilon_{0}}(x, y)= & \frac{1}{2 \pi \imath} \int_{\rho^{\prime} C_{1}} \sum_{n=-\infty}^{\infty} a_{n, \varepsilon_{0}}(x) z_{1}^{n} \omega_{\varepsilon}\left(y, z_{1}\right) \\
& +\frac{1}{2 \pi \imath} \int_{\rho^{\prime} C_{2}} \sum_{n=-\infty}^{\infty} a_{n, \varepsilon_{0}}(x) \varepsilon^{n} / z_{2}^{n} \omega_{\varepsilon}\left(y, z_{2}\right) \\
= & -\sum_{n} \frac{1}{2 \pi \imath} \int_{\rho^{\prime} C_{2}} a_{n, \varepsilon_{0}}(x)\left(\varepsilon^{n} / z_{2}^{n}\right) \omega_{\varepsilon}\left(y, z_{2}\right) \\
& +\sum_{n} \frac{1}{2 \pi i} \int_{\rho^{\prime} C_{2}} a_{n, \varepsilon_{0}}(x)\left(\varepsilon_{0}^{n} / z_{2}^{n}\right) \omega_{\varepsilon}\left(y, z_{2}\right) \\
= & \sum_{n}\left(\varepsilon_{0}^{n}-\varepsilon^{n}\right) a_{n, \varepsilon_{0}}(x) \frac{1}{2 \pi \imath} \int_{\rho^{\prime} C_{2}} w_{\varepsilon}\left(y, z_{2}\right) / z_{2}^{n} .
\end{aligned}
$$

Lemma 3 shows that the estimate

$$
\omega_{\varepsilon}\left(y, z_{2}\right)=O(1) \quad y \in\left(S_{1} \backslash \rho U_{1}\right) \cup\left(S_{2} \backslash \rho U_{2}\right), \quad z_{2} \in \rho^{\prime} C_{2}
$$

holds uniformly. In addition, the identity

$$
\varepsilon^{n}-\varepsilon_{0}^{n}=n \varepsilon_{0}^{n-1}\left(\varepsilon-\varepsilon_{0}\right)+\left(\varepsilon-\varepsilon_{0}\right)^{2} R_{n}
$$

where

$$
R_{n}=\left\{\begin{array}{cc}
\frac{1}{2 \pi i} \int_{|z|=r_{1}} \frac{z^{n} d z}{\left(z-\varepsilon_{0}\right)^{2}(z-\varepsilon)}, & n \geqq 0 \\
-\frac{1}{2 \pi i} \int_{|z|=r_{2}} \frac{z^{n} d z}{\left(z-\varepsilon_{0}\right)^{2}(z-\varepsilon)}, & n<0
\end{array}\right.
$$

with $0<r_{2}<\left|\varepsilon_{0}\right|<r_{1}$, implies that

$$
R_{n}= \begin{cases}O\left(\left(\sqrt{\left.\varepsilon_{0}\right)^{n}}\right)\right. & n \rightarrow+\infty, \\ O\left(\left(\varepsilon_{0} \sqrt{\rho^{\prime}}\right)^{n}\right) & n \rightarrow-\infty .\end{cases}
$$

Thus the estimate

$$
\omega_{\varepsilon}(x, y)-\omega_{\varepsilon_{0}}(x, y)=O\left(\varepsilon-\varepsilon_{0}\right) \quad\left(\varepsilon \rightarrow \varepsilon_{0}\right)
$$

holds uniformly for $x, y \in\left(S_{1} \backslash \rho U_{1}\right) \cup\left(S_{2} \backslash \rho U_{2}\right)$. Since $\rho$ is arbitrary, (19) also holds for $x, y \in\left(S_{1} \backslash \rho^{\prime \prime} U_{1}\right) \cup\left(S_{2} \backslash \rho^{\prime \prime} U_{2}\right)$ with $\left|\varepsilon_{0}\right|^{1 / 2}<\rho^{\prime \prime}<\rho^{\prime}$. Therefore, if (19) with $\rho$ replaced by $\rho^{\prime \prime}$ is substituted in (17), it follows easily that

$$
\begin{aligned}
\omega_{\varepsilon}(x, y) & -\omega_{\varepsilon_{0}}(x, y) \\
& =\sum_{n}\left(\varepsilon_{0}^{n}-\varepsilon^{n}\right) a_{n, \varepsilon_{0}}(x) \frac{1}{2 \pi \imath} \int_{\rho^{\prime} C_{2}} \omega_{\varepsilon_{0}}\left(y, z_{2}\right) / z_{2}^{n}+O\left(\left(\varepsilon-\varepsilon_{0}\right)^{2}\right) \\
& =\sum_{n}\left(\varepsilon^{n}-\varepsilon_{0}^{n}\right) a_{n, \varepsilon_{0}}(x) \frac{\varepsilon_{0}^{-n}}{2 \pi \imath} \int_{\rho^{\prime} C_{1}} z_{1}^{n} \omega_{\varepsilon_{0}}\left(y, z_{1}\right)+O\left(\left(\varepsilon-\varepsilon_{0}\right)^{2}\right)
\end{aligned}
$$




$$
\begin{aligned}
& =\sum_{n}\left(\varepsilon^{n}-\varepsilon_{0}^{n}\right) \varepsilon_{0}^{-n} a_{n, \varepsilon_{0}}(x)(-n) a_{-n, \varepsilon_{0}}(y)+O\left(\left(\varepsilon-\varepsilon_{0}\right)^{2}\right) \\
& =-\frac{\varepsilon-\varepsilon_{0}}{\varepsilon_{0}} \sum_{n} n^{2} a_{n, \varepsilon_{0}}(x) a_{-n, \varepsilon_{0}}(y)+O\left(\left(\varepsilon-\varepsilon_{0}\right)^{2}\right) .
\end{aligned}
$$

Here we used (18), (19) and Cauchy's integral theorem.

Theorems 2 and 3 clearly show that $\omega_{\varepsilon}(x, y)$ is holomorphic in $\varepsilon$ at $\varepsilon=0$. To obtain the Taylor expansions of $\omega_{\varepsilon}(x, y)$ with respect to $\varepsilon$, the following observations are in order: let

$$
\alpha_{k l}^{(j)}=\frac{1}{2 \pi \imath} \int_{\rho C_{\jmath}} a_{k}^{(j)}\left(z_{\jmath}\right) / z_{\jmath}^{l} \quad(\jmath=1,2 ; k, l=1,2, \cdots)
$$

with $0<\rho<1$ and set, for $|\varepsilon|<\rho^{2}$,

$$
\omega_{\varepsilon}(x, y)=\sum_{n=0}^{\infty} \varepsilon^{n} \Omega_{n}(x, y), \quad x, y \in\left(S_{1} \backslash \rho U_{1}\right) \cup\left(S_{2} \backslash \rho U_{2}\right)
$$

where $\Omega_{o}(x, y) \equiv \omega_{o}(x, y)$ defined before. From (11) and (14) it follows easily that

$$
\alpha_{k l}^{(j)}=\beta_{k-1, l-1}^{(j)} / k \quad(\jmath=1,2 ; k, l=1,2, \cdots) .
$$

Thus the symmetry $\beta_{k l}^{(j)}=\beta_{l k}^{(j)}$ implies

$$
k \alpha_{k l}^{(j)}=l \alpha_{l k}^{(j)} \quad(j=1,2 ; k, l=1,2, \cdots) .
$$

$\Omega_{n}(x, y)(n=1,2, \cdots)$ are bilinear holomorphic differentials on $\left(S_{1} \backslash \rho U_{1}\right) \cup\left(S_{2} \backslash \rho U_{2}\right)$, since the singularities of $\omega_{s}(x, y)$ are cancelled out by those of $\Omega_{0}(x, y)$.

With the above preparation, the following theorem concerning the variational formulas of any order will now be demonstrated.

THEOREM 4. The $n$-th order variational coefficients $\Omega_{n}(x, y)(n=1,2, \cdots)$ are given by: for $j=1,2$ and $0<\rho<1$,

where

$$
\Omega_{n}(x, y)=\left\{\begin{array}{c}
\sum_{h, k=1}^{n+k \leq n} \Omega_{n, j j}^{h k} a_{h}^{(j)}(x) a_{k}^{(j)}(y), \quad x, y \in S_{\jmath} \backslash \rho U_{\jmath}, \\
\sum_{h, k=1}^{n+k \leq n} \Omega_{n, j j^{\prime}}^{h k} a_{h}^{(j)}(x) a_{k}^{\left(j^{\prime}\right)}(y)-n a_{n}^{(j)}(x) a_{n}^{\left(j^{\prime}\right)}(y), \\
x \in S_{\jmath} \backslash \rho U_{\jmath}, y \in S_{j^{\prime}} \backslash \rho U_{j^{\prime}}
\end{array}\right.
$$

$$
\begin{aligned}
& \Omega_{n, j j}^{h k}=h \sum \alpha_{h t_{1}}^{\left(j^{\prime}\right)} \alpha_{t_{1} t_{2}}^{(j)} \alpha_{t_{2} t_{3}}^{\left(j^{\prime}\right)} \cdots \alpha_{t_{2 s}}^{\left(j^{\prime}\right)}, \\
& \Omega_{n, j j}^{h k}=-h \sum \alpha_{h t_{1}}^{\left(j^{\prime}\right)} \alpha_{t_{1} t_{2}}^{(j)} \alpha_{t_{2} t_{3}}^{\left(j^{\prime}\right)} \cdots \alpha_{t_{2 s+1} k}^{(j)}
\end{aligned}
$$

with summation taken over all integral vectors $\left(t_{j}\right)$ such that

$$
n-h-k=\sum_{j=1}^{2 s} t_{\jmath}, \quad t_{j} \geqq 1, s \geqq 0, s \in \boldsymbol{Z}
$$


and

$$
n-h-k=\sum_{j=1}^{2 s+1} t_{\jmath}, \quad t_{j} \geqq 1, s \geqq 0, s \in \boldsymbol{Z}
$$

respectively.

Proof. From Lemma 4, (5), (21) and Cauchy's integral theorem, it is seen

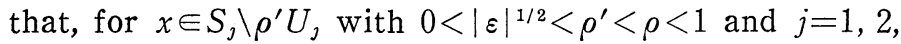

$$
\begin{aligned}
\sum_{n=1}^{\infty} \varepsilon^{n} \Omega_{n}(x, y) & =\frac{1}{2 \pi \imath} \int_{\rho^{\prime} C_{j}}\left(\sum_{n=1}^{\infty} a_{n}^{(j)}(x) z_{j}^{n}\right) \omega_{\varepsilon}(y, z) \\
& =-\frac{1}{2 \pi \imath} \int_{\rho^{\prime} C_{j^{\prime}}}\left(\sum_{n=1}^{\infty} \varepsilon^{n} a_{n}^{(j)}(x) z_{j^{\prime}}^{-n}\right)\left(\sum_{m=0}^{\infty} \varepsilon^{m} \Omega_{m}\left(y, z_{j^{\prime}}\right)\right) \\
& =-\sum_{n=1}^{\infty} \sum_{m=0}^{\infty} \varepsilon^{n+m} a_{n}^{(j)}(x) \frac{1}{2 \pi \imath} \int_{\rho^{\prime} C_{j^{\prime}}} \Omega_{m}\left(y, z_{j^{\prime}}\right) / z_{j^{\prime}}^{n} .
\end{aligned}
$$

Compairing coefficients of like powers of $\varepsilon$, we obtain

$$
\Omega_{n}(x, y)=-\sum_{h=1}^{n} a_{h}^{(j)}(x) \frac{1}{2 \pi \imath} \int_{\rho^{\prime} C_{j^{\prime}}} \Omega_{n-h}\left(y, z_{j^{\prime}}\right) / z_{j^{\prime}}^{h}
$$

for $n=1,2, \cdots, x \in S_{j} \backslash \rho^{\prime} U_{3}(\jmath=1,2)$ and $y \in\left(S_{1} \backslash \rho^{\prime} U_{1}\right) \cup\left(S_{2} \backslash \rho^{\prime} U_{2}\right)$. Since $\rho^{\prime}$ is arbitrary, the repeated use of (26) gives

$$
\Omega_{n}(x, y)=\left\{\begin{aligned}
\sum_{h, k=1}^{n+k \leq n} a_{h}^{(j)}(x) a_{k}^{(j)}(y) \frac{1}{(2 \pi i)^{2}} \int_{\rho^{\prime} C_{j^{\prime} \times \rho^{\prime} C_{j^{\prime}}}} \Omega_{n-h-k}(z, w) / z^{h} w^{k} \\
\text { for } x, y \in S \backslash \rho U_{\jmath}, \\
\sum_{n, k=1} a_{h}^{(j)}(x) a_{k}^{\left(j^{\prime}\right)}(y) \frac{1}{(2 \pi i)^{2}} \int_{\rho^{\prime} C_{j^{\prime} \times \rho^{\prime} C_{\jmath}}} \Omega_{n-h-k}(z, w) / z^{h} w^{k} \\
-n a_{n}^{(j)}(x) a_{n}^{\left(j^{\prime}\right)}(y) \text { for } \quad x \in S_{\jmath} \backslash \rho U_{\jmath}, y \in S_{j^{\prime}} \backslash \rho U_{j^{\prime}} .
\end{aligned}\right.
$$

For it is easily seen that $\Omega_{o} \equiv \omega_{0}$ satisfies

$$
\frac{1}{2 \pi i} \int_{\rho^{\prime} C_{j^{\prime}}} \Omega_{0}\left(y, z_{j^{\prime}}\right) / z_{j^{\prime}}^{n}= \begin{cases}0 & y \in S_{\jmath} \backslash \rho U_{j} \\ n a_{n}^{\left(j^{\prime}\right)}(y) & y \in S_{j^{\prime}} \backslash \rho U_{j^{\prime}}\end{cases}
$$

by definitions (11) and (13). On setting (for $n=1,2, \cdots ; h, k \geqq 1, h+k \leqq n$; $j=1,2$ )

$$
\begin{aligned}
& \Omega_{n, j j}^{h k}=\frac{1}{(2 \pi i)^{2}} \int_{\rho C_{j^{\prime}} \times \rho C_{j^{\prime}}} \Omega_{n-h-k}(z, w) / z^{h} w^{k}, \\
& \Omega_{n, j j^{\prime}}^{h k}=\frac{1}{(2 \pi i)^{2}} \int_{\rho C_{j^{\prime} \times \rho C}} \Omega_{n-h-k}(z, w) / z^{h} w^{k},
\end{aligned}
$$

it remains only to show that the formulas (25) and $(25)^{\prime}$ hold. But this is easy, if one notes the following recurrence formulas for $\Omega_{n, j j}^{h k}$ and $\Omega_{n, j j^{\prime}}^{h k}$ obtained 
by substituting (24) in the integrand $\Omega_{n-h-k}(z, w)$ on the right hand side of (28): for $n=1,2, \cdots ; h, k \geqq 1, h+k \leqq n ; \jmath=1,2$,

$$
\begin{aligned}
\Omega_{n, j j}^{h k} & = \begin{cases}\sum_{p, q=1}^{p+q \leq n-h-k} \Omega_{n-h-k, j^{\prime} j^{\prime}}^{p q} \alpha_{p h}^{\left(j^{\prime}\right)} \alpha_{q k}^{\left(j^{\prime}\right)} & h+k<n \\
h \alpha_{h k}^{\left(j^{\prime}\right)} & h+k=n\end{cases} \\
\Omega_{n, j j^{\prime}}^{h k} & =\sum_{p, q=1}^{p+q \leq n-h-k} \Omega_{n-h-k, j^{\prime} j}^{p q} \alpha_{p h}^{\left(j^{\prime}\right)} \alpha_{q k}^{(j)}-(n-h-k) \alpha_{n-h-k, h}^{\left(j^{\prime}\right)} \alpha_{n-h-k, k}^{(j)} .
\end{aligned}
$$

By induction on $n$, it turns out that (25) and (25)' are the direct consequences of (23), (29) and (29)'. This completes the proof of Theorem 4.

Clearly, (23), (25) and (25)' show that the important quantities $\Omega_{n, i j}^{h k}$ have the symmetry:

$$
\Omega_{n, j j}^{h k}=\Omega_{n, j j}^{k h}, \quad \Omega_{n, j j^{\prime}}^{h k}=\Omega_{n, j^{\prime} J}^{k h}
$$

for $n=1,2, \cdots ; h, k \geqq 1, h+k \leqq n ; \jmath=1,2$.

Remark. The coefficients $\Omega_{n, j j}^{h k}$ satisfy the following identity : for $n=1,2, \cdots$; $h, k=1,2, \cdots, h+k \leqq n ; \jmath=1,2$,

$$
(n-h-k) \Omega_{n, j j}^{h k}=\sum_{\nu=1}^{n-1} \sum_{l=1}^{l+h \leqq \nu} \sum_{m=1}^{m+k \leqq n-\nu}\left(\alpha_{l m}^{(i)}+\alpha_{m l}^{(j)}\right) \Omega_{\nu, j j}^{h l} \Omega_{n-\nu, j \jmath}^{k m} .
$$

To show (31) we calculate $a_{n, \varepsilon}(x)(n= \pm 1, \pm 2, \cdots)$ explicitly by using Theorem 4. For $x \in S_{1} \backslash U_{1}$ termwise integration gives

$$
\int^{z_{1}} \omega_{\varepsilon}(\cdot, x)=\text { const. }+\sum_{n=1}^{\infty} a_{n}^{(1)}(x) z_{1}^{n}+\sum_{n=1}^{\infty} \sum_{h, k=1}^{h+k \leq n} \varepsilon^{n} \Omega_{n, 11}^{h k} a_{h}^{(1)}(x) \int^{z_{1}} a_{k}^{(1)} .
$$

By (20) the integrals on the right hand side have expansions

$$
\int^{z_{1}} a_{k}^{(1)}=-\frac{1}{k z_{1}^{k}}+\text { const. }+\sum_{i=1}^{\infty} \frac{\alpha_{k l}^{(1)}}{l} z_{1}^{l}, \quad k=1,2, \cdots .
$$

Thus, from the definition (11), it is seen that $a_{n, \varepsilon}(x)$ is given by: for $x \in S_{1} \backslash U_{1}$ and $n=1,2, \cdots$,

$$
\begin{aligned}
& a_{-n, \varepsilon}(x)=-\frac{1}{n} \sum_{m, h=1}^{n+n \leq m} \varepsilon^{m} \Omega_{m, 11}^{h n} a_{h}^{(1)}(x), \\
& a_{n, \varepsilon}(x)=a_{n}^{(1)}(x)+\frac{1}{n} \sum_{m=1}^{\infty} \sum_{n, k=1}^{n+k \leq m} \varepsilon^{m} \alpha_{k n}^{(1)} \Omega_{m, 11}^{h k} a_{h}^{(1)}(x) .
\end{aligned}
$$

Hence it follows from Theorems 3 and 4 that, for $x, y \in S_{1} \backslash U_{1}$,

$$
\begin{aligned}
\sum_{n=1}^{\infty} n \varepsilon^{n} \Omega_{n}(x, y)= & \sum_{n=1}^{\infty}\left(\sum \varepsilon^{m} \Omega_{m, 11}^{h n} a_{h}^{(1)}(x)\right)\left(n a_{n}^{(1)}(y)+\sum \varepsilon^{m} \alpha_{k n}^{(1)} \Omega_{m, 11}^{h k} a_{h}^{(1)}(y)\right) \\
& +\sum_{n=1}^{\infty}\left(\sum \varepsilon^{m} \Omega_{m}^{h n} a_{11 h}^{(1)}(y)\right)\left(n a_{n}^{(1)}(x)+\sum \varepsilon^{m} \alpha_{k n}^{(1)} \Omega_{m, 11}^{h k} a_{h}^{(1)}(x)\right) .
\end{aligned}
$$


Compairing coefficients of $\varepsilon^{n}$ in the above expansions, we find an expression of $\Omega_{n}(x, y)$ different from (24), which, together with (24), easily implies (31). Similar identities will also be obtained by choosing $x$ or $y \in S_{2} \backslash U_{2}$ in the above calculations.

Now that Theorem 4 is obtained, it will be possible to derive variational formulas for any meromorphic differentials on $S_{\varepsilon}$ which is holomorphic on $P_{\varepsilon}$. However, instead of computing complicated formulas for the general case, we restrict ourselves to the case of the normalized differentials of the first and the third kind.

Let $\omega_{a-b}^{(j)}$ (resp. $\omega_{a-b, \varepsilon}$ ) be the normalized differential of the third kind on $S_{\text {, }}\left(\right.$ resp. $S_{\varepsilon}$ ) with simple poles of residue 1 and -1 at $a, b \in S_{\jmath}$ (resp. $S_{\varepsilon}$ ) respectively. Then the Riemann bilinear relation gives

$$
\begin{aligned}
& v_{k}(x)= \begin{cases}\int_{B_{k}} \omega_{1}(\cdot, x) & x \in S_{1}, 1 \leqq k \leqq g_{1} \\
\int_{B_{k}} \omega_{2}(\cdot, x) & x \in S_{2}, g_{1}+1 \leqq k \leqq g,\end{cases} \\
& \omega_{a-b}^{(j)}(x)=\int_{b}^{a} \omega_{j}(\cdot, x) \quad x \in S_{\jmath}, \jmath=1,2
\end{aligned}
$$

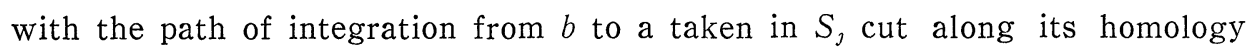
basis. For notational convenience, let the following expansion holds in terms of the pinching coordinates:

$$
\int_{p_{j}}^{z} \eta=\sum_{n=1}^{\infty} \gamma_{n}^{(j)}[\eta] z_{j}^{n}, \quad\left|z_{j}\right|<1
$$

where $\eta$ is any differential holomorphic on $U_{\jmath}(\jmath=1,2)$. Thus (11) may be rewritten as

$$
\gamma_{n}^{(j)}\left[\omega_{j}(\cdot, x)\right]=a_{n}^{(j)}(x) .
$$

Furthermore, let us write for short

$$
\gamma_{n}^{(j)}\left[v_{k}\right]=\gamma_{n k}^{(j)}, \quad \gamma_{n}^{(j)}\left[\omega_{a-b}^{(j)}\right]=\gamma_{n}^{(j)}(a, b), \quad \gamma_{n}^{(j)}\left[\omega_{a-p_{j}}^{(j)}+d z_{j} / z_{\jmath}\right]=\gamma_{n}^{(j)}(a) .
$$

Analogous to (32), the Riemann bilinear relation again gives

$$
\int_{B_{k}} a_{n}^{(j)}(\cdot)=\gamma_{n k}^{(j)}, \quad \int_{b}^{a} a_{n}^{(j)}(\cdot)=\gamma_{n}^{(j)}(a, b) .
$$

If (21) is integrated term by term along the cycle $B_{k}(\varepsilon)=B_{k}$, an expansion of $v_{k, \varepsilon}$ will be obtained at once in view of (32) and (35). Indeed, this is legitimate since $B_{k}$ is contained in the region where Theorem 4 is valid.

COROLLARY 1. The normalized differentials of the first kind on $S_{\varepsilon}$ have expansions near $\varepsilon=0$ : for $i=1,2, \cdots, g_{1}$ and $0<\rho<1$, 


$$
v_{i, \varepsilon}(x)= \begin{cases}v_{i}(x)+\varepsilon^{2} \beta_{00}^{(2)} v_{i}\left(p_{1}\right) \omega_{1}\left(x, p_{1}\right)+O\left(\varepsilon^{3}\right) & x \in S_{1} \backslash \rho U_{1} \\ -\varepsilon v_{i}\left(p_{1}\right) \omega_{2}\left(x, p_{2}\right)+O\left(\varepsilon^{2}\right) & x \in S_{2} \backslash \rho U_{2}\end{cases}
$$

where $\beta_{00}^{(2)}$ is the constant defined by (14) and the estrmates $O\left(\varepsilon^{2}\right)$ and $O\left(\varepsilon^{3}\right)$ are uniform.

More precisely for $\imath=1, \cdots, g$ and $x \in\left(S_{1} \backslash \rho U_{1}\right) \cup\left(S_{2} \backslash \rho U_{2}\right)$

$$
v_{i, \varepsilon}(x)=\sum_{n=0}^{\infty} \varepsilon^{n}\left(v_{i}\right)_{n}(x)
$$

where, for $i=1, \cdots, g_{1}$ and $n=1,2, \cdots$,

$$
\begin{aligned}
& \left(v_{i}\right)_{0}(x)= \begin{cases}v_{i}(x) & x \in S_{1}, \\
0 & x \in S_{2},\end{cases} \\
& \left(v_{i}\right)_{n}(x)= \begin{cases}\sum_{n, k=1}^{n+k \leq n} \Omega_{n, 11}^{h k} \gamma_{h i}^{(1)} a_{k}^{(1)}(x) & x \in S_{1} \backslash \rho U_{1}, \\
\sum_{n, k=1}^{h+k \leq n} \Omega_{n, 12}^{k h} \gamma_{h i}^{(1)} a_{k}^{(2)}(x)-n \gamma_{n \imath}^{(1)} a_{n}^{(2)}(x) & x \in S_{2} \backslash \rho U_{2} .\end{cases}
\end{aligned}
$$

For $i=g_{1}+1, \cdots, g$, similar formulas are obtained by symmetry.

If (37) is integrated term by term along $B_{k}$ once again, a variational formula for the period matrix for $S_{\varepsilon}$, denoted by $\tau_{\varepsilon}$, is obtained.

COROLlARY 2. The period matrix $\tau_{\varepsilon}$ has an expansion near $\varepsilon=0$

$$
\tau_{\varepsilon}=\left(\begin{array}{cc}
\tau_{1} & 0 \\
0 & \tau_{2}
\end{array}\right)-\varepsilon\left(\begin{array}{cc}
0 & { }^{t} R_{1} R_{2} \\
{ }^{t} R_{2} R_{1} & 0
\end{array}\right)+O\left(\varepsilon^{2}\right)
$$

where $\tau_{1}$ and $\tau_{2}$ are the period matrices for $S_{1}$ and $S_{2}$ respectively, and

More precisely:

$$
\begin{aligned}
& R_{1}=\left(v_{1}\left(p_{1}\right), \cdots, v_{g_{1}}\left(p_{1}\right)\right) \in \boldsymbol{C}^{g_{1}}, \\
& R_{2}=\left(v_{g_{1}+1}\left(p_{2}\right), \cdots, v_{g}\left(p_{2}\right)\right) \in \boldsymbol{C}^{g_{2}} .
\end{aligned}
$$

$$
\tau_{\varepsilon}=\sum_{n=0}^{\infty} \varepsilon^{n} \tau_{n}
$$

where $\tau_{n}=\left(\tau_{n, \imath j}\right)_{i, j=1}^{g}$ and, for $n=1,2, \cdots$,

$$
\tau_{n, \imath j}= \begin{cases}\sum_{n, k=1}^{n+k \leqq n} \Omega_{n, 11}^{h k} \gamma_{h \imath}^{(1)} \gamma_{k J}^{(1)} & 1 \leqq i, j \leqq g_{1}, \\ \sum_{n, k=1}^{h+k \leqq n} \Omega_{n, 22}^{h k} \gamma_{h \imath}^{(2)} \gamma_{k J}^{(2)} & g_{1}+1 \leqq \imath, j \leqq g,\end{cases}
$$




$$
\tau_{n, \imath j}=\tau_{n, j i}=\sum_{h, k=1}^{h+k \leqq n} \Omega_{n, 12}^{h k} \gamma_{h \imath}^{(1)} \gamma_{k J}^{(2)}-n \gamma_{n \imath}^{(1)} \gamma_{n j}^{(2)} \quad \text { for } \quad 1 \leqq \imath \leqq g_{1}, g_{1}+1 \leqq \jmath \leqq g .
$$

Here $\gamma_{h i}^{(j)}(j=1,2)$ is the constant defined by (33) and (34).

Similarly, variational formulas for $\omega_{a-b, \varepsilon}$ are obtained if both $a$ and $b \in S_{j} \backslash \rho U_{\text {, with }} 0<\rho<1(j=1,2)$. On the other hand, if $a \in S_{j} \backslash \rho U_{j}$ and $b \in S_{j^{\prime}} \backslash$ $\rho U_{j^{\prime}}(j=1,2)$, such a simple method as above fails immediately since the path of integration must across the pinched region. In this case, however, we can proceed as follows: analogous to Lemma $4, \omega_{a-b, \varepsilon}(x)$ is given in terms of $\omega_{\varepsilon}(x, y)$ by

$$
\begin{aligned}
\omega_{a-b, \varepsilon}(x)-\omega_{a-b, o}(x)= & \frac{1}{2 \pi \imath} \int_{\rho C_{\jmath}}\left(\int_{p_{\jmath}}^{z}\left(\omega_{a-p_{\jmath}}^{(j)}+d z_{j} / z_{\jmath}\right)\right) \omega_{\varepsilon}(x, z) \\
& -\frac{1}{2 \pi \imath} \int_{\rho C_{j^{\prime}}}\left(\int_{p_{j^{\prime}}}^{z}\left(\omega_{b^{\prime}-p_{j^{\prime}}}^{\left(j^{\prime}\right)}+d z_{j^{\prime}} / z_{j^{\prime}}\right)\right) \omega_{\varepsilon}(x, z)
\end{aligned}
$$

where $a \in S_{j} \backslash \rho U_{\jmath}, b \in S_{j^{\prime}} \backslash \rho U_{j^{\prime}}, x \in\left(S_{1} \backslash \rho U_{1}\right) \cup\left(S_{2} \backslash \rho U_{2}\right)$ and

$$
\omega_{a-b, o}(x)= \begin{cases}\omega_{a-p_{1}}^{(1)}(x) & x \in S_{1}, \\ \omega_{p_{2}-b}^{(2)}(x) & x \in S_{2} .\end{cases}
$$

(41) follows from a similar reasoning as in the proof of Lemma 4, so that the proof may be omitted. If the expansion (21) is substituted in the right hand side of (41), the desired variational formula is obtained from Theorem 4 by termwise integration. The results are summarized as follows.

COROLLARY 3. The normalized differential of the third kind $\omega_{a-b, \varepsilon}(x)$ has an expansion near $\varepsilon=0$ :

(i) for $a, b \in S_{j} \backslash \rho U_{\jmath}$,

$$
\omega_{a-b, \varepsilon}(x)= \begin{cases}\omega_{a-b}^{(j)}(x)+\varepsilon^{2} \beta_{00}^{\left(j^{\prime}\right)} \omega_{a-b}^{(j)}\left(p_{j}\right) \omega_{j}\left(x, p_{\jmath}\right)+O\left(\varepsilon^{3}\right) & x \in S_{\jmath} \backslash \rho U_{\jmath}, \\ -\varepsilon w_{a-b}^{(j)}\left(p_{j}\right) \omega_{j^{\prime}}\left(x, p_{j^{\prime}}\right)+O\left(\varepsilon^{2}\right) & x \in S_{j^{\prime}} \backslash \rho U_{j^{\prime}},\end{cases}
$$

(ii) for $x, a \in S_{j} \backslash \rho U_{j}$, and $b \in S_{j^{\prime}} \backslash \rho U_{j^{\prime}}$,

$$
\omega_{a-b, \varepsilon}(x)=\omega_{a-p_{j}}^{(j)}(x)+\varepsilon d_{j^{\prime}}(b) \omega_{j}\left(x, p_{j}\right)+O\left(\varepsilon^{2}\right)
$$

where, in terms of the pinching coordinates,

$$
d_{j}(a)=\lim _{z \rightarrow p_{j}}\left[\omega_{a-p_{j}}(z)+1 / z_{j}(z)\right] \in C \quad(j=1,2)
$$

and all the estimates $O\left(\varepsilon^{2}\right), O\left(\varepsilon^{3}\right)$ are uniform.

More precisely:

$$
\omega_{a-b, \varepsilon}(x)=\sum_{n=0}^{\infty} \varepsilon^{n}\left(\omega_{a-b}\right)_{n}(x) \quad x \in\left(S_{1} \backslash \rho U_{1}\right) \cup\left(S_{2} \backslash \rho U_{2}\right)
$$


where, for $n=1,2, \cdots$,

(i)' for $a, b \in S_{\jmath} \backslash \rho U_{\jmath}$,

$$
\left(\omega_{a-b}\right)_{n}(x)=\left\{\begin{array}{l}
\sum_{h, k \leq 1}^{h+k \leq n} \Omega_{n, j j}^{h k} \gamma_{h}^{(j)}(a, b) a_{k}^{(j)}(x), \quad x \in S_{\jmath} \backslash \rho U_{\jmath}, \\
\sum_{h, k=1}^{h+k \leq n} \Omega_{n, j j^{\prime}}^{h k} \gamma_{h}^{(j)}(a, b) a_{k}^{\left(j^{\prime}\right)}(x)-n \gamma_{n}^{(j)}(a, b) a_{n}^{\left(j^{\prime}\right)}(x), \quad x \in S_{j^{\prime}} \backslash \rho U_{j^{\prime}},
\end{array}\right.
$$

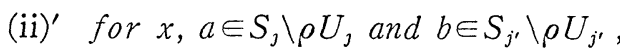

$$
\left(\omega_{a-b}\right)_{n}(x)=\sum_{h, k=1}^{h+k \leq n}\left\{\Omega_{n, j j}^{h k} \gamma_{h}^{(j)}(a)-\Omega_{n, j^{\prime} j}^{h k} \gamma_{h}^{\left(j^{\prime}\right)}(b)\right\} a_{k}^{(j)}(x)+n \gamma_{n}^{\left(j^{\prime}\right)}(b) a_{n}^{(j)}(x) .
$$

Here $\gamma_{h}^{(j)}(a, b)$ and $\gamma_{h}^{(j)}(a)$ are the constants defined by (33) and (34).

On account of the importance of the coefficients $\alpha_{n k}^{(j)}$, we make mention of the close connection between the differentials $a_{n}^{(j)}(x)(n=1,2, \cdots)$ and the Faber polynomials.

For convenience, let us omit the letter " $j$ " in our notation and write

$$
S=S_{\jmath}, \quad U=U_{\jmath}, \quad a_{n}(x)=a_{n}^{(j)}(x), \quad \text { etc. }
$$

The local coordinate $z: U \rightarrow \Delta$ is, on the other hand, regarded as a univalent mapping $\phi(t)=z^{-1}(1 / t):\{t ;|t|>1\} \rightarrow S$. In the case where $S=\hat{C}$ (the Riemann sphere) and $p=\infty(\in \hat{C}), \phi$ is a complex-valued function and the expansion (11) reduces to

$$
\sum_{n=1}^{\infty} a_{n}(x) t^{-n}=\int_{\infty}^{t} \frac{\phi^{\prime}(s) d s}{(\phi(s)-x)^{2}}=\frac{1}{x-\phi(t)} .
$$

Recall that a generating function for the Faber polynomials $p_{n}(n=1,2, \cdots)$ belonging to $\phi$ is given by

$$
\frac{x \phi^{\prime}(t)}{\phi(t)-x}=1+\frac{p_{1}(x)}{t}+\frac{p_{2}(x)}{t^{2}}+\cdots
$$

[2]. From (47) and (48) it is easily seen that

$$
p_{n}(x)-p_{n}\left(x_{0}\right)=-n \int_{x_{0}}^{x} a_{n}(t) d t \quad(n=1,2, \cdots) .
$$

In view of this identity, it is natural to call the Abelian integrals $\mathscr{I}_{n}(x)$ $=-n \int^{x} a_{n} d t$ the Faber integrals belonging to a local coordinate $z$, which agree, up to a constant, the Faber polynomials if $S=\hat{\boldsymbol{C}}$ and $p=\infty$. From (20) $\mathscr{I}_{n}{ }^{\circ} \phi$ $(n=1,2, \cdots)$ has an expansion 


$$
\mathscr{F}_{n}{ }^{\circ} \phi(w)=w^{n}+\text { const. }-\sum_{k=1}^{\infty} \frac{n}{k} \alpha_{n k} w^{-k}, \quad|w|>1 .
$$

With this analogy, $\alpha_{n k}(n, k=1,2, \cdots)$ may be called the generalized Faber coefficients and the equation (23) corresponds to the classical Grunsky law of symmetry [2]. Furthermore, a generalization of Golusin inequality is obtained from a straightforward analog of area theorems as follows.

THEOREM 5. Let $\left\{x_{n}\right\}$ be an arbitrary sequence of complex numbers. Then the coefficients $\alpha_{n k}$ satisfy

$$
\sum_{k=1}^{\infty} \frac{1}{k}\left|\sum_{n=1}^{N} x_{n} \alpha_{n k}\right|^{2} \leqq \sum_{n=1}^{N} \frac{1}{n}\left|x_{n}\right|^{2}, \quad(N=1,2, \cdots)
$$

Equality holds for a non-zero sequence $\left\{x_{n}\right\}$ if and only if the complement of the image of $\phi$ in $S$ has areal measure zero.

Proof. Let us evaluate the norm of $\sum_{n=1}^{N} x_{n} a_{n}(x)$ on $S \backslash \rho U$ with $0<\rho<1$. The Riemann bilinear relation and (50) give

$$
\begin{aligned}
& \frac{1}{2 \pi}\left\|\sum_{n=1}^{N} x_{n} a_{n}(\cdot)\right\|_{S \backslash \rho U}^{2} \\
& =-\frac{1}{2 \pi i} \iint_{S \backslash \rho U} \sum_{n=1}^{N} x_{n} a_{n}(\cdot) \wedge \overline{\sum_{n=1}^{N} x_{n} a_{n}(\cdot)} \\
& =\frac{1}{2 \pi i} \int_{\rho C} \overline{\left(\int^{X} \sum_{n=1}^{N} x_{n} a_{n}(\cdot)\right)} \sum_{n=1}^{N} x_{n} a_{n}(x) \\
& =\frac{1}{2 \pi i} \int_{|w|=1 / \rho} \overline{\sum_{n=1}^{N} \frac{x_{n}}{n} \mathscr{F}_{n} \circ \phi(w) d}\left[\sum_{n=1}^{N} \frac{x_{n}}{n} \mathscr{F}_{n} \circ \phi(w)\right] \\
& =\sum_{n=1}^{N} \frac{\left|x_{n}\right|^{2}}{n} \rho^{-2 n}-\sum_{k=1}^{\infty} \frac{1}{k}\left|\sum_{n=1}^{N} x_{n} \alpha_{n k}\right|^{2} \rho^{2 k} .
\end{aligned}
$$

Letting $\rho \rightarrow 1$, we have

$$
\frac{1}{2 \pi}\left\|\sum_{n=1}^{N} x_{n} a_{n}(\cdot)\right\|_{S \backslash U}^{2}=\sum_{n=1}^{N} \frac{1}{n}\left|x_{n}\right|^{2}-\sum_{k=1}^{\infty} \frac{1}{k}\left|\sum_{n=1}^{N} x_{n} \alpha_{n k}\right|^{2},
$$

which obviously implies Theorem 5 . The equality statement is a direct consequence of the linear independence of $a_{n}(x)^{\prime} s$.

When $S=\hat{\boldsymbol{C}}$ the inequality (51) has been already obtained by Jenkins [5], Milin [7] and Pommerenke [9]. Applying the Cauchy inequality to (51), we have at once a version of Grunsky inequality : let $\left\{x_{n}\right\}$ be an arbitrary sequence of complex numbers. Then,

$$
\left|\sum_{k, n=1}^{N} \beta_{n-1, k-1} x_{n} x_{k}\right| \leqq \sum_{n=1}^{N} n\left|x_{n}\right|^{2} \quad(N=1,2, \cdots)
$$


where $\beta_{n k}(n, k=0,1, \cdots)$ are the coefficients of the expansion (14). (Note the identity (22).) Equality condition is the same as in Theorem 5 .

In particular the important quantities $\beta_{00}^{(j)}$ appearing in Theorem 2 and Corollaries 3 and 4 satisfy

$$
\left|\beta_{00}^{(j)}\right| \leqq 1 \quad(\jmath=1,2) .
$$

Remark. Schiffer and Spencer have proved an inequality more general than (52) in their book [10] where they generalized, to the case of finite bordered Riemann surfaces, Grunsky's necessary and sufficient condition for the univalence of an analytic function defined on the exterior of the unit circle. Since (51) implies (52), their theorem 5.5.3. [10, p. 168] can be restated. For the sake of completeness, we record this fact as a

COROLlary. Let $\phi$ map a neighborhood of $0 \in \Delta$ conformally into a neighborhood of $p=\phi(0) \in S$. Using a local coordinate $\phi^{-1}$ around $p$, one may calculate the series expansion (14). Then $\phi$ can be extended over $\Delta$ to give an analytic imbedding of $\Delta$ into $S$ if and only if the inequalities (51) hold for every sequence $\left\{x_{n}\right\}$ of complex numbers.

\section{Pinching along a non-zero homology cycle.}

Here, the notation and the definitions in the previous sections are used unless otherwise stated.

Let $S$ be a compact Riemann surface of genus $g$ and choose coordinates $z_{1}: U_{1} \rightarrow \Delta$ and $z_{2}: U_{2} \rightarrow \Delta$ in disjoint neighborhoods $U_{1}$ and $U_{2}$ of two points $p_{1}, p_{2} \in S$. Again, a family of compact Riemann surfaces $\left\{S_{\varepsilon} ; \varepsilon \in \boldsymbol{C}, 0<|\varepsilon|<1\right\}$ formed from $S$ is constructed by identifying $U_{1}$ and $U_{2}$ under the condition (5). $S_{\varepsilon}$ is a compact Riemann surface of genus $g+1$ while the pinched region $P_{\varepsilon}=S_{\varepsilon} \backslash\left(S \backslash\left(U_{1} \cup U_{2}\right)\right)$ is usually identified by the pinching coordinates $z_{1}$ and $z_{2}$ with the annulus $|\varepsilon|<|z|<1$ as before. To choose some canonical homology basis for $S$, let $A_{1}(\varepsilon), B_{1}(\varepsilon), \cdots, A_{g}(\varepsilon), B_{g}(\varepsilon)$ simply be a canonical basis $A_{1}, B_{1}, \cdots, A_{g}, B_{g}$ for $S$ lying in $S \backslash\left(U_{1} \cup U_{2}\right)$. In addition let $A_{g+1}(\varepsilon)=\rho C_{2}$ with any $\rho$ satisfying $|\varepsilon|<\rho<1$ and let $B_{g+1}(\varepsilon)$ be any path from $z_{1}^{-1}(\sqrt{\varepsilon})$ to $z_{2}^{-1}(\sqrt{\varepsilon})$ lying within $S \backslash\left(|\varepsilon| U_{1} \cup|\varepsilon| U_{2}\right)$ cut along the homology basis for $S$.

Corresponding to Theorem 1 , the following analogous theorem holds with trivial modification, so that proof will be omitted.

THEOREM 1'. Let $\Omega$ be a meromorphic differential on $S$ which is holomorphic on $U_{1}$ and $U_{2}$ except for possible simple poles at $p_{1}$ and $p_{2}$ with residues $-\alpha$ and $\alpha$ respectively, and let

$$
\alpha_{n}^{(j)}=\gamma_{n}^{(j)}\left[\Omega-(-1)^{\jmath} \alpha d z_{j} / z_{\jmath}\right]
$$

for $j=1,2$ and $n=1,2, \cdots$. Then there exists a meromorphic differential $\Omega_{\varepsilon}$ on $S_{\varepsilon}$ which is holomorphic on $P_{\varepsilon}$ with the same singularities as $\Omega$ on $S \backslash\left(U_{1} \cup U_{2}\right)$, satısfying 
$(6)^{\prime}$

$$
\left\|\Omega_{\varepsilon}-\Omega\right\|_{S \backslash\left(\rho U_{1} \cup \rho U_{2}\right)}^{2} \leqq \pi \sum_{n=1}^{\infty} n\left(\left|\alpha_{n}^{(1)}\right|^{2}+\left|\alpha_{n}^{(2)}\right|^{2}\right) \frac{|\rho \varepsilon|^{2 n}}{\rho^{4 n}-|\varepsilon|^{2 n}}
$$

with $|\varepsilon|^{1 / 2}<\rho<1$.

On applying Theorem $1^{\prime}$ and using the identity analogous to Lemma 4

$$
\omega_{\varepsilon}(x, y)-\omega(x, y)=\frac{1}{2 \pi \imath} \int_{\rho C_{1}+\rho C_{2}}\left(\int^{z} \omega(\cdot, x)\right) \omega_{\varepsilon}(z, y)
$$

for $x, y \in S \backslash\left(\rho U_{1} \cup \rho U_{2}\right)$ with $|\varepsilon|^{1 / 2}<\rho<1$, it is now easy to deduce such variational formulas as in Theorems 2,3 and 4 by a method similar to the one used in section 2. For instance, the uniform boundedness of $v_{\jmath, \varepsilon}(\jmath=1, \cdots$, $g+1)$ will be shown immediately by choosing

$$
\Omega= \begin{cases}v_{\jmath} & \text { if } \jmath=1, \cdots, g \\ \omega_{p_{2}-p_{1}} & \text { if } \quad \jmath=g+1\end{cases}
$$

and applying Theorem $1^{\prime}$. (This is the reason why simple poles at $p_{1}$ and $p_{2}$ must be permitted for the $\Omega$ in Theorem $1^{\prime}$ as the singularity.)

Now the main results in this section will be summarized almost without proof in the form of a theorem and corollaries. In order to state these, let us define

$$
\alpha_{p l}^{s_{\jmath}}=\frac{1}{2 \pi \imath} \int_{\rho C_{\jmath}} a_{p}^{(s)}\left(z_{j}\right) / z_{\jmath}^{l} \quad(s, \jmath=1,2 ; p, l=1,2, \cdots)
$$

which, corresponding to (20), are important to express the variational coefficients. Again, it follows the symmetry:

$$
l \alpha_{l m}^{j k}=m \alpha_{m l}^{k j} \quad(j, k=1,2 ; l, m=1,2, \cdots)
$$

THEOREM 6. $\omega_{\varepsilon}(x, y)$ has an expansion near $\varepsilon=0:$ for $x, y \in S /\left(\rho U_{1} \cup \rho U_{2}\right)$ with $0<\rho<1$,

$$
\omega_{\varepsilon}(x, y)=\omega(x, y)-\varepsilon\left[\omega\left(x, p_{1}\right) \omega\left(y, p_{2}\right)+\omega\left(x, p_{2}\right) \omega\left(y, p_{1}\right)\right]+O\left(\varepsilon^{2}\right)
$$

where the estimate $O\left(\varepsilon^{2}\right)$ is uniform.

More precisely: for $|\varepsilon|^{1 / 2}<\rho<1$

$$
\omega_{\varepsilon}(x, y)=\sum_{n=0}^{\infty} \varepsilon^{n} \Omega_{n}(x, y) \quad x, y \in S \backslash\left(\rho U_{1} \cup \rho U_{2}\right)
$$

where $\Omega_{o}(x, y)=\omega(x, y)$ and, for $n=1,2, \cdots$,

$$
\Omega_{n}(x, y)=\sum_{\jmath, k=1}^{2} \sum_{l, m=1}^{l+m \leq n} \Omega_{n, j k}^{i m} a_{l}^{(j)}(x) a_{m}^{(k)}(y)-\sum_{j=1}^{2} n a_{n}^{(j)}(x) a_{n}^{\left(j^{\prime}\right)}(y) .
$$

The coefficients $\Omega_{n, j k}^{l m}$ are given by: for $l, m, n=1,2, \cdots(l+m \leqq n)$ and $\jmath, k=1,2$,

$$
\Omega_{n, j k}^{l m}=l \sum(-1)^{d} \alpha_{l t_{1}}^{j^{\prime} s_{1}} \alpha_{t_{1} t_{2}}^{s_{1} s_{2}} \alpha_{t_{2} t_{3}}^{s_{2} s_{3}} \cdots \alpha_{t_{d}^{m}}^{s d^{\prime} k^{\prime}}
$$


with summation taken over all vectors $\left(s_{p}\right)$ and $\left(t_{q}\right) \in Z^{d}$ such that

$$
n-l-m=\sum_{\jmath=1}^{d} t_{\jmath}, \quad t_{j} \geqq 1, s_{\jmath}=1,2, d \geqq 0 .
$$

Instead of (29), the recurrence formula for $\Omega_{n, j k}^{l m}$ is given by

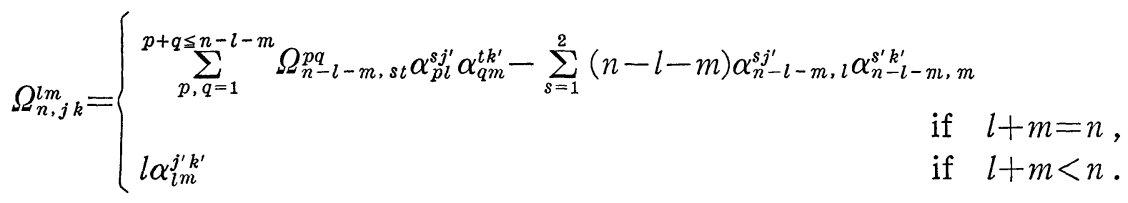

By induction on $n$, (25)" is verified from $(23)^{\prime}$ and $(29)^{\prime}$ as before.

Integration of $(21)^{\prime}$ along the cycle $B_{j}(\varepsilon)(\jmath=1, \cdots, g)$ immediately yields

COROLlary 4. For $i=1, \cdots, g, v_{i, \varepsilon}(x)$ has an expansion near $\varepsilon=0$ : for $x \in S \backslash\left(\rho U_{1} \cup \rho U_{2}\right)$ with $0<\rho<1$,

$$
v_{i, \varepsilon}(x)=v_{i}(x)-\varepsilon\left[v_{i}\left(p_{2}\right) \omega\left(x, p_{1}\right)+v_{i}\left(p_{1}\right) \omega\left(x, p_{2}\right)\right]+O\left(\varepsilon^{2}\right)
$$

where the estimate $O\left(\varepsilon^{2}\right)$ is uniform.

More precisely:

$$
v_{i, \varepsilon}(x)=\sum_{n=0}^{\infty} \varepsilon^{n}\left(v_{i}\right)_{n}(x) \quad x \in S \backslash\left(\rho U_{1} \cup \rho U_{2}\right)
$$

where $\left(v_{i}\right)_{0}(x)=v_{i}(x)$ and, for $n=1,2, \cdots$,

$$
\left(v_{i}\right)_{n}(x)=\sum_{j, k=1}^{2} \sum_{l, m=1}^{l+m \leq n} \Omega_{n, j k}^{l m} \gamma_{i i}^{(j)} a_{m}^{(k)}(x)-\sum_{j=1}^{2} n \gamma_{n i}^{(j)} a_{n}^{\left(j^{\prime}\right)}(x)
$$

with $\gamma_{n k}^{(j)}=\gamma_{n}^{(j)}\left[v_{k}\right]$.

On the other hand, Theorem 6 and the identity

$$
\begin{aligned}
v_{g+1, \varepsilon}(x)-\omega_{p_{2}-p_{1}}(x)= & \frac{1}{2 \pi i} \int_{\rho C_{1}}\left(\int^{z}\left(\omega_{p_{2}-p_{1}}+d z_{1} / z_{1}\right)\right) \omega_{\varepsilon}(z, x) \\
& +\frac{1}{2 \pi i} \int_{\rho C_{2}}\left(\int^{z}\left(\omega_{p_{2}-p_{1}}-d z_{2} / z_{2}\right)\right) \omega_{\varepsilon}(z, x)
\end{aligned}
$$

for $x \in S \backslash\left(\rho U_{1} \cup \rho U_{2}\right)$ with $|\varepsilon|^{1 / 2}<\rho<1$ give

COROLlaRY 5. $v_{g+1, \varepsilon}(x)$ has an expansion near $\varepsilon=0$ : for $x \in S \backslash\left(\rho U_{1} \cup \rho U_{2}\right)$

$$
v_{g+1, \varepsilon}(x)=\omega_{p_{2}-p_{1}}(x)-\varepsilon\left[\gamma_{2} \omega\left(x, p_{1}\right)+\gamma_{1} \omega\left(x, p_{2}\right)\right]+O\left(\varepsilon^{2}\right)
$$

where the estimate $O\left(\varepsilon^{2}\right)$ is uniform and the constants

$$
\gamma_{j}=\lim _{x \rightarrow p_{\jmath}}\left[\omega_{p_{2}-p_{1}}(x)-(-1)^{\jmath} d z_{j}(x) / z_{\jmath}(x)\right] \quad(\jmath=1,2)
$$

are evaluated in terms of the pinching coordinates. 
More precisely:

$$
v_{g+1, \varepsilon}(x)=\sum_{n=0}^{\infty} \varepsilon^{n}\left(v_{g+1}\right)_{n}(x) \quad x \in S \backslash\left(\rho U_{1} \cup \rho U_{2}\right)
$$

where $\left(v_{g+1}\right)_{0}(x)=\omega_{p_{2}-p_{1}}(x)$ and, for $n=1,2, \cdots$,

$$
\left(v_{g+1}\right)_{n}(x)=\sum_{j, k=1}^{2} \sum_{l, m=1}^{l+m \leq n} \Omega_{n, j k}^{l m} \gamma_{l}^{(j)} a_{m}^{(k)}(x)-\sum_{j=1}^{2} n \gamma_{n}^{(j)} a_{n}^{\left(j^{\prime}\right)}(x)
$$

with $\gamma_{n}^{(j)}=\gamma_{n}^{(j)}\left[\omega_{p_{2}-p_{1}}-(-1)^{\jmath} d z_{j} / z_{\jmath}\right]$.

Let

$$
\tau_{\varepsilon}=\left(\begin{array}{c:c}
\tau_{\imath \jmath, \varepsilon} & \sigma_{\imath, \varepsilon} \\
\hdashline \sigma_{\jmath, \varepsilon} & \sigma_{\varepsilon}
\end{array}\right)^{g} \in G L(g+1, \boldsymbol{C})
$$

be the period matrix for $S_{\varepsilon}$ with respect to a canonical basis $A_{1}(\varepsilon), B_{1}(\varepsilon), \cdots$, $A_{g+1}(\varepsilon), B_{g+1}(\varepsilon)$. From Corollaries 4 and 5 , it is easy to calculate the Taylor expansion of $\tau_{\varepsilon}$ at $\varepsilon=0$ except for the $(g+1, g+1)$-element $\sigma_{\varepsilon}$ for which the path of integration $B_{g+1}(\varepsilon)$ must across the pinched region. The next lemma shows that $\sigma_{\varepsilon}$ can be expressed through the line integrals whose paths of integration avoid the pinched region.

LEMMA 5. For $\varepsilon \in \boldsymbol{C}$ and $\rho \in \boldsymbol{R}$ satısfying $0<|\varepsilon|^{1 / 2}<\rho<1 / 2$, the following identity holds:

$$
\begin{aligned}
\sigma_{\varepsilon}=\ln 4 \varepsilon+\int_{z_{1}^{-1}(1 / 2)}^{z_{2}^{-1}(1 / 2)} \omega_{p_{2}-p_{1}} & +\frac{1}{2 \pi \imath} \int_{\rho C_{1}}\left(\int_{z_{1}^{-1}(1 / 2)}^{z}\left(\omega_{p_{2}-p_{1}}+d z_{1} / z_{1}\right)\right) v_{g+1, \varepsilon}(z) \\
& +\frac{1}{2 \pi i} \int_{\rho C_{2}}\left(\int_{z_{2}^{-1}(1 / 2)}^{z}\left(\omega_{p_{2}-p_{1}}-d z_{2} / z_{2}\right)\right) v_{g+1, \varepsilon}(z) .
\end{aligned}
$$

(The proper choice of the logarithm depends on the path chosen to define the cycle $B_{g+1}(\varepsilon)$.)

Proof. Cauchy's integral theorem and the bilinear relation give

$$
\int_{\rho C_{1}+\rho C_{2}}\left(\int_{z_{1}^{-1}(1 / 2)}^{z}\left(v_{g+1, \varepsilon}-\omega_{p_{2}-p_{1}}\right)\right) v_{g+1, \varepsilon}(z)=0 \text {. }
$$

Hence

$$
\begin{aligned}
\int_{z_{1}^{-1}(1 / 2)}^{z_{2}^{-1}(1 / 2)}\left(v_{g+1, \varepsilon}-\omega_{p_{2}-p_{1}}\right) & \\
= & \frac{1}{2 \pi i} \int_{\rho C_{1}}\left(\int_{z_{1}^{-1}(1 / 2)}^{z}\left(\omega_{p_{2}-p_{1}}+\frac{d z_{1}}{z_{1}}\right)\right) v_{g+1, \varepsilon}(z) \\
& +\frac{1}{2 \pi i} \int_{\rho C_{2}}\left(\int_{z_{2}^{-1}(1 / 2)}^{z}\left(\omega_{p_{2}-p_{1}}-\frac{d z_{2}}{z_{2}}\right)\right) v_{g+1, \varepsilon}(z)
\end{aligned}
$$




$$
\begin{aligned}
& -\frac{1}{2 \pi i} \int_{\rho C_{1}}\left(\int_{z_{1}^{-1}(1 / 2)}^{z}\left(v_{g+1, \varepsilon}+\frac{d z_{1}}{z_{1}}\right)\right) v_{g+1, \varepsilon}(z) \\
& -\frac{1}{2 \pi \imath} \int_{\rho C_{2}}\left(\int_{z_{2}^{-1}(1 / 2)}^{z}\left(v_{g+1, \varepsilon}-\frac{d z_{2}}{z_{2}}\right)\right) v_{g+1, \varepsilon}(z) .
\end{aligned}
$$

On the other hand, a change of parameter by using $z_{1} z_{2}=\varepsilon$ yields

$$
\begin{aligned}
& \frac{1}{2 \pi \imath} \int_{\rho C_{1}}\left(\int_{z_{1}^{-1}(1 / 2)}^{z}\left(v_{g+1, \varepsilon}+\frac{d z_{1}}{z_{1}}\right)\right) v_{g+1, \varepsilon}(z) \\
& \quad=-\frac{1}{2 \pi \imath} \int_{\rho C_{2}}\left(\int_{z_{2}^{-1}(2 \varepsilon)}^{z}\left(v_{g+1, \varepsilon}-\frac{d z_{2}}{z_{2}}\right)\right) v_{g+1, \varepsilon}(z) .
\end{aligned}
$$

Therefore

$$
\begin{aligned}
\int_{z_{1}^{-1}(1 / 2)}^{z^{-1}(2 \varepsilon)} v_{g+1, \varepsilon}-\int_{z_{1}^{-1}(1 / 2)}^{z_{2}^{-1}(1 / 2)} \omega_{p_{2}-p_{1}}-\int_{z_{2}^{-1}(1 / 2)}^{z_{2}^{-1}(2 \varepsilon)} d z_{2} / z_{2} \\
=\frac{1}{2 \pi \imath} \int_{\rho C_{1}}\left(\int_{z_{1}^{-1}(1 / 2)}^{z}\left(\omega_{p_{2}-p_{1}}+\frac{d z_{1}}{z_{1}}\right)\right) v_{g+1, \varepsilon}(z) \\
+\frac{1}{2 \pi \imath} \int_{\rho C_{2}}\left(\int_{z_{2}^{-1}(1 / 2)}^{z}\left(\omega_{p_{2}-p_{1}}-\frac{d z_{2}}{z_{2}}\right)\right) v_{g+1, \varepsilon}(z) .
\end{aligned}
$$

Note that the path of integration from $z_{1}^{-1}(1 / 2)$ to $z_{2}^{-1}(2 \varepsilon)$ can be identified with $B_{g+1}(\varepsilon)$, so that the proof is completed.

From the above lemma, it is seen that the constant term in the expansion (60) is given by :

$$
\begin{aligned}
& \ln 4+\int_{z_{1}^{-1}(1 / 2)}^{z^{-1}(1 / 2)} \omega_{p_{2}-p_{1}}+\frac{1}{2 \pi \imath} \int_{\rho C_{1}}\left(\int_{z_{1}^{-1}(1 / 2)}^{z}\left(\omega_{p_{2}-p_{1}}+\frac{d z_{1}}{z_{1}}\right)\right) \omega_{p_{2}-p_{1}}(z) \\
&+\frac{1}{2 \pi i} \int_{\rho C_{2}}\left(\int_{z_{2}^{-1}(1 / 2)}^{z}\left(\omega_{p_{2}-p_{1}}-\frac{d z_{2}}{z_{2}}\right)\right) \omega_{p_{2}-p_{1}}(z) \\
&=\ln 4+\int_{z_{1}^{-1}(1 / 2)}^{z^{-1}(1 / 2)} \omega_{p_{2}-p_{1}}-\int_{z_{1}^{-1}(1 / 2)}^{z_{1}^{-1}(0)}\left(\omega_{p_{2}-p_{1}}+\frac{d z_{1}}{z_{1}}\right)+\int_{z_{2}^{-1}(1 / 2)}^{z_{2}^{-1}(0)}\left(\omega_{p_{2}-p_{1}}-\frac{d z_{2}}{z_{2}}\right) .
\end{aligned}
$$

This, in turn, is seen to be equal to the constant

$$
\lim _{x \rightarrow 0}\left[\int_{z_{1}^{-1}(x)}^{z_{2}^{-1}(x)} \omega_{p_{2}-p_{1}}-2 \ln x\right] .
$$

Corollaries 4, 5 and Lemma 5 give immediately the expansion of the period matrix.

COROLlaRY 6. Let $\gamma_{1}, \gamma_{2}, \gamma_{n}^{(j)}$ and $\gamma_{n k}^{(j)}$ be defined as in Corollaries 4 and 5 . Then the period matrix for $S_{\varepsilon}$ has an expansion 


$$
\tau_{\varepsilon}=\left(\begin{array}{ll}
\tau_{\imath \jmath}+\varepsilon \sigma_{\imath \jmath} & a_{i}+\varepsilon \sigma_{\imath} \\
a_{\jmath}+\varepsilon \sigma_{\jmath} & \ln \varepsilon+c_{0}+c_{1} \varepsilon
\end{array}\right)+O\left(\varepsilon^{2}\right)
$$

where $\left(\tau_{\imath j}\right)_{i, j=1}^{g}$ is the period matrix for $S, a_{\imath}=\int_{p_{1}}^{p_{2}} v_{\imath}$,

$$
\sigma_{\imath j}=-\left(v_{i}\left(p_{1}\right) v_{\jmath}\left(p_{2}\right)+v_{i}\left(p_{2}\right) v_{\jmath}\left(p_{1}\right)\right), \quad \sigma_{\imath}=-\left(\gamma_{1} v_{i}\left(p_{2}\right)+\gamma_{2} v_{i}\left(p_{1}\right)\right),
$$

$c_{0}=\lim _{x \rightarrow 0}\left[\int_{z_{1}^{-1}(x)}^{z^{-1}(x)} \omega_{p_{2}-p_{1}}-2 \ln x\right]$ and $c_{1}=-2 \gamma_{1} \gamma_{2}$.

More precisely.

$$
\tau_{\imath \jmath, \varepsilon}=\sum_{n=0}^{\infty} \varepsilon^{n}\left(\tau_{\imath j}\right)_{n} \quad \imath, \jmath=1,2, \cdots, g
$$

where $\left(\tau_{\imath j}\right)_{0}=\tau_{\imath j}$ and, for $n=1,2, \cdots$

$$
\begin{gathered}
\left(\tau_{\imath j}\right)_{n}=\sum_{s, t=1}^{2} \sum_{l, m=1}^{l+m \leqq n} \Omega_{n, s t}^{l m} \gamma_{l i}^{(s)} \gamma_{m J}^{(t)}-\sum_{s=1}^{2} n \gamma_{m i}^{(s)} \gamma_{n J}^{\left(s^{\prime}\right)} . \\
\sigma_{\imath, \varepsilon}=\sum_{n=0}^{\infty} \varepsilon^{n}\left(\sigma_{\imath}\right)_{n} \quad i=1, \cdots, g
\end{gathered}
$$

where $\left(\sigma_{\imath}\right)_{0}=\int_{B_{\imath}} \omega_{p_{2}-p_{1}}=\int_{p_{1}}^{p_{2}} v_{i}$ and, for $n=1,2, \cdots$,

$$
\begin{gathered}
\left(\sigma_{\imath}\right)_{n}=\sum_{s, t=1}^{2} \sum_{l, m=1}^{l+m \leq n} \Omega_{n, s t}^{l m} \gamma_{l}^{(s)} \gamma_{m i}^{(t)}-\sum_{s=1}^{2} n \gamma_{n}^{(s)} \gamma_{n \imath}^{\left(s^{\prime}\right)} . \\
\sigma_{\varepsilon}=\ln \varepsilon+\sum_{n=0}^{\infty} \varepsilon^{n}(\sigma)_{n}
\end{gathered}
$$

where $(\sigma)_{0}=c_{0}$ and, for $n=1,2, \cdots$,

$$
(\sigma)_{n}=\sum_{s, t=1}^{2} \sum_{l, m=1}^{l+m \leq n} \Omega_{n, s t}^{l m} \gamma_{l}^{(s)} \gamma_{m}^{(t)}-2 n \gamma_{n}^{(1)} \gamma_{n}^{(2)}
$$

From Theorem 6 , it is also possible to derive a variational formula for the prime form $E(x, y)$. For the basic properties of $E(x, y)$ the reader may consult [3, Chap. 2].

Since the multipliers of $E(x, y)$ and $E_{\varepsilon}(x, y)$ (the prime form for $S_{\varepsilon}$ ) along the cycles $\rho C_{1}$ and $\rho C_{2}$ are both equal to 1 (c.f. [3]), we can choose a single-valued branch of $\ln \left(E_{\varepsilon}(x, y) / E(x, y)\right)$ over $S \backslash\left(\rho U_{1} \cup \rho U_{2}\right)$ canonically dissected so as to satisfy

$$
\lim _{x, y \rightarrow q} \ln \left(E_{\varepsilon}(x, y) / E(x, y)\right)=0
$$

for any $q \in S \backslash\left(\rho U_{1} \cup \rho U_{2}\right)$ [3, Corollary 2.5]. From now on, all paths of integration are taken within a fixed canonical dissection containing $\rho U_{1}$ and $\rho U_{2}$. With this agreement we have 
Corollary 7. In $E_{\varepsilon}(x, y)$ has an expansion: for $x, y \in S \backslash\left(\rho U_{1} \cup \rho U_{2}\right)$ with $|\varepsilon|^{1 / 2}<\rho<1$,

$$
\ln E_{\varepsilon}(x, y)=\ln E(x, y)-\varepsilon \omega_{y-x}\left(p_{1}\right) \omega_{y-x}\left(p_{2}\right)+O\left(\varepsilon^{2}\right)
$$

where the estimate $O\left(\varepsilon^{2}\right)$ is uniform.

More precisely.

$$
\begin{aligned}
\ln E_{\varepsilon}(x, y)= & \ln E(x, y)-\sum_{n=1}^{\infty} n \varepsilon^{n} \int_{x}^{y} a_{n}^{(1)} \int_{x}^{y} a_{n}^{(2)} \\
& +\frac{1}{2} \sum_{n=1}^{\infty} \varepsilon^{n} \sum_{j, k=1}^{2} \sum_{l, m=1}^{l+m \leqq n} \Omega_{n, j k}^{l m} \int_{x}^{y} a_{l}^{(j)} \int_{x}^{y} a_{m}^{(k)} .
\end{aligned}
$$

Proof. First we note the identity [3, Corollary 2.6]: for $x, y \in S$,

$$
\omega(x, y)=\frac{\partial^{2}}{\partial x \partial y} \ln E(x, y) d x d y \text {. }
$$

To prove (66), set

$$
\begin{aligned}
F(x, y)= & \ln \left(E_{\varepsilon}(x, y) / E(x, y)\right)+\sum_{n=1}^{\infty} n \varepsilon^{n} \int_{x}^{y} a_{n}^{(1)} \int_{x}^{y} a_{n}^{(2)} \\
& -\frac{1}{2} \sum_{n=1}^{\infty} \varepsilon^{n} \sum_{j, k=1}^{2} \sum_{l, m=1}^{l+m \leq n} \Omega_{n, j k}^{l m} \int_{x}^{y} a_{l}^{(j)} \int_{x}^{y} a_{m}^{(k)}
\end{aligned}
$$

and consider $\frac{\partial^{2}}{\partial x \partial y} F(x, y)$. (67) and Theorem 6 show

$$
\frac{\partial^{2}}{\partial x \partial y} F(x, y)=0 \text {. }
$$

On account of the symmetry $F(x, y)=F(y, x)$ (c. f. [3]), it is seen that $F(x, y)$ has the form

$$
F(x, y)=h(x)+h(y)
$$

where $h(x)$ is single-valued and holomorphic on $S \backslash\left(\rho U_{1} \cup \rho U_{2}\right)$ canonically dissected, since $a_{n}^{(j)}(x)(j=1,2$ and $n=1,2, \cdots)$ has no residues at $p_{1}$ and $p_{2}$. (64) implies that $F(x, x)=0$ or $h(x)=0$, so that $F(x, y)=0$. This gives (66) while (65) is proved by recalling the identity (c.f. [3])

$$
\omega_{a-b}(x)=\int_{b}^{a} \omega(\cdot, x)
$$

and the proof is completed.

Remark. Let $g(x, y)$ be the Green's function on a planar regular region $D$. Then it can be verified that

$$
g(x, y)=\ln \left|\frac{E(x, \bar{y})}{E(x, y)}\right| \quad x, y \in D
$$


where $E(x, y)$ is the prime form for the double of $D$ with respect to a suitable canonical homology basis and $\bar{y}$ is the conjugate point of $y \in D$. (69) shows that Robin's constant $c(x)$ is given by

$$
c(x)=\ln |E(x, \bar{x})| .
$$

By using the representations (69) and (70), Corollary 7 will yield variational formulas for $g(x, y)$ and $c(x)$, but we do not enter into these calculations.

\section{Examples.}

To guarantee the validity of our formulas, we consider here two cases where $\omega_{\varepsilon}(x, y)$ can be calculated easily by other methods.

ExAmple 1. Let $S_{1}$ and $S_{2}$ be the extended complex plane $\hat{\boldsymbol{C}}$. Then the fundamental normalized differential $\omega_{j}(x, y)$ is given by

$$
\omega_{j}(x, y)=\frac{d x d y}{(x-y)^{2}} \quad(\jmath=1,2) .
$$

If $\left|\kappa_{j}\right|<1$, the function $\phi_{j}(z)=\frac{1}{z}+\kappa_{j} z$ maps conformally the unit disk $\Delta$ onto $U_{j} \subset \hat{\boldsymbol{C}}$ with $\phi_{j}(0)=\infty(j=1,2)$. Hence it is possible to take $\phi_{j}^{-1}$ as a coordinate $z_{\jmath}: U_{j} \rightarrow \Delta$ on $S_{\jmath}$ centered at $p_{\jmath}=\infty \quad(\jmath=1,2)$. Since $S_{\varepsilon}$ has genus zero, it is well-known that, for any fixed $x \in S_{1} \backslash U_{1}$, there exists a conformal mapping $f_{\varepsilon}: S_{\varepsilon} \rightarrow \hat{\boldsymbol{C}}$ satisfying $f_{\varepsilon}(x)=\infty$. To calculate $\omega_{\varepsilon}(x, y)$ on $S_{\varepsilon}$, we shall first study the mapping $f_{\varepsilon}$ itself. Let $f_{j}$ be the restriction of $f_{\varepsilon}$ to $S_{j} \backslash|\varepsilon| U_{j}(j=1,2)$, and assume without loss of generality that $f_{1}$ is holomorphic on $S_{1} \backslash|\varepsilon| U_{1}$ except for a simple pole at $x$ with residue 1 . In view of the equation (5), $f_{1}$ and $f_{2}$ must satisfy

$$
f_{1}\left(\phi_{1}(z)\right)=f_{2}\left(\phi_{2}(\varepsilon / z)\right) \quad \text { for } \quad|\varepsilon|<|z|<1 .
$$

From the functional equations

$$
\phi_{\mathrm{J}}(z)=\phi_{j}\left(\frac{1}{\kappa_{j} z}\right) \quad(\jmath=1,2)
$$

(for simplicity assume $\kappa_{1} \kappa_{2} \neq 0$ here, as in the sequel), it follows that (1) is extended meromorphically to the function $F(z)$ which is now defined on $0<|z|<\infty$. By (72) and (73) $F(z)$ satisfies

$$
F(z)=F\left(\frac{1}{\kappa_{1} z}\right)=F\left(\frac{\kappa_{2} \varepsilon^{2}}{z}\right),
$$

so that

$$
F(z)=F\left(\kappa_{1} \kappa_{2} \varepsilon^{2} z\right), \quad 0<|z|<\infty .
$$

Thus $F\left(e^{z}\right)$ becomes a doubly periodic function with periods $2 \pi i$ and 
$\alpha\left(=\ln \kappa_{1} \kappa_{2} \varepsilon^{2}\right)$ which is holomorphic except for simple poles at $z \equiv \ln \beta$ and $z \equiv-\ln \kappa_{1} \beta$ (mod periods) with residues $\left(\kappa_{1} \beta-\frac{1}{\beta}\right)^{-1}$ and $-\left(\kappa_{1} \beta-\frac{1}{\beta}\right)^{-1}$ respectively; here $\beta$ denotes a number satisfying $\phi_{1}(\beta)=x$. As is seen from the theory of elliptic functions, $F\left(e^{z}\right)$ has an explicit representation:

$$
F\left(e^{z}\right)=\left(\kappa_{1} \beta-\frac{1}{\beta}\right)^{-1}\left[\zeta(z-\ln \beta)-\zeta\left(z+\ln \kappa_{1} \beta\right)\right]+\text { const. }
$$

where $\zeta(z)=\zeta(z ; 2 \pi \imath, \alpha)$ is the Weierstrassian zeta-function. On the other hand, it is well-known (see $[4, \mathrm{p}, 477])$ that $\zeta(z)$ has a series expansion given by

$$
\zeta(z)=\eta z+\frac{1}{2} \frac{e^{z}+1}{e^{z}-1}+\sum_{n=1}^{\infty} \frac{h^{n} e^{-z}}{1-h^{n} e^{-z}}-\sum_{n=1}^{\infty} \frac{h^{n} e^{z}}{1-h^{n} e^{z}}
$$

where

$$
h=e^{\alpha}=\kappa_{1} \kappa_{2} \varepsilon^{2} \quad \text { and } \quad \eta=\frac{1}{2 \pi \imath}(\zeta(z+2 \pi i)-\zeta(z)) .
$$

Hence, if (75) is substituted in (74), we have

$$
\begin{aligned}
& \left(\kappa_{1} \beta-\frac{1}{\beta}\right) F(z)=\text { const. }+\frac{1}{2} \frac{z+\beta}{z-\beta}+\sum_{n=1}^{\infty} \frac{h^{n}}{z-h^{n} \beta}-\sum_{n=1}^{\infty} \frac{h^{n} z}{\beta-h^{n} z} \\
& -\frac{1}{2} \frac{\kappa_{1} \beta z+1}{\kappa_{1} \beta z-1}-\sum_{n=1}^{\infty} \frac{h^{n}}{\kappa_{1} \beta z-h^{n}}+\sum_{n=1}^{\infty} \frac{h^{n} \kappa_{1} \beta z}{1-h^{n} \kappa_{1} \beta z} \\
& =\text { const. }+\frac{\kappa_{1} \beta-\beta^{-1}}{\phi_{1}(z)-x}+\sum_{n=1}^{\infty} \sum_{d=1}^{\infty} h^{n d} \kappa_{1}^{-d}\left(\kappa_{1}^{d} \beta^{d}-\beta^{-d}\right)\left(z^{-d}+\kappa_{1}^{d} z^{d}\right) \text {. }
\end{aligned}
$$

Observe that, for fixed $x \in S_{1} \backslash U_{1}, \omega_{\varepsilon}\left(x, \phi_{1}(z)\right)$ is given by

$$
\omega_{\varepsilon}\left(x, \phi_{1}(z)\right)=-F^{\prime}(z) / \phi_{1}^{\prime}(z) .
$$

Thus, if (76) is differentiated, it follows that

$$
\omega_{\varepsilon}\left(x, \phi_{1}(z)\right)=\frac{1}{\left(\phi_{1}(z)-x\right)^{2}}-\sum_{n=1}^{\infty} \sum_{d=1}^{\infty} d \kappa_{1}^{-d} h^{n d} \frac{\left(\kappa_{1} \beta\right)^{d}-\beta^{-d}}{\kappa_{1} \beta-\beta^{-1}} \frac{\left(\kappa_{1} z\right)^{d}-z^{-d}}{\kappa_{1} z-z^{-1}}
$$

for $x, \phi_{1}(z) \in S_{1} \backslash U_{1}$. To show that (77) agrees with the expansion given by Theorem 4, let us determine the differentials $a_{n}(x)$ and the coefficients $\Omega_{n, 11}^{h k}$. By (71) and the definition (11), $a_{n}^{(1)}(x)(n=1,2, \cdots)$ are given by the expansion

$$
\sum_{n=1}^{\infty} a_{n}^{(j)}(x) z^{n}=\frac{1}{x-\phi_{j}(z)} \quad(j=1,2),
$$

and thus, after easy calculation, it is seen that

$$
a_{n}^{(1)}(x)=\frac{\left(\kappa_{1} \beta\right)^{d}-\beta^{-d}}{\kappa_{1} \beta-\beta^{-1}}, \quad a_{n}^{(1)}\left(\phi_{1}(z)\right)=\frac{\left(\kappa_{1} z\right)^{d}-z^{-d}}{\kappa_{1} z-z^{-1}} .
$$

If (78) is substituted in (77), we conclude finally 


$$
\omega_{\varepsilon}(x, y)=\frac{1}{(x-y)^{2}}-\sum_{n=1}^{\infty} h^{n} \sum_{d 1 n} d \kappa_{1}^{-d} a_{d}^{(1)}(x) a_{d}^{(1)}(y)
$$

with $h=\kappa_{1} \kappa_{2} \varepsilon^{2}$. On the other hand, one verifies that

$$
\alpha_{n m}=-\delta_{n m} \kappa_{j}^{n} \quad(\jmath=1,2)
$$

with $\delta_{n m}$ the Kronecker $\delta$, since the expansion (14) has the form

$$
\phi_{j}^{*} \omega_{j}(x, y)=\frac{1}{(x-y)^{2}}-\frac{\kappa_{j}}{\left(1-\kappa_{j} x y\right)^{2}}=\frac{1}{(x-y)^{2}}-\sum_{n=0}^{\infty}(n+1) \kappa_{j}^{n+1} x^{n} y^{n} .
$$

From (80) and (25) the variational coefficients $\Omega_{n, 11}^{h k}$ are easily calculated. The result is that $\Omega_{n, 11}^{h k}$ 's all vanish except when $n$ is even and $h=k$. In the exceptional case, $\Omega_{2 n, 11}^{d d}$ is given by

$$
\Omega_{2 n, 11}^{a d}= \begin{cases}-d\left(\kappa_{1} \kappa_{2}\right)^{n} \kappa_{1}^{-d} & \text { if } d \mid n, \\ 0 & \text { otherwise. }\end{cases}
$$

Hence (79) completely agrees with our Theorem 4.

EXAMPLE 2. With the same notation as in section 3, we set:

$$
S=\hat{\boldsymbol{C}}, U_{1}=\{z ;|z|<r\}, U_{2}=\{z ;|z|>R\}, p_{1}=0, p_{2}=\infty, z_{1}=z / r, z_{2}=R / z
$$

where $r$ and $R$ are numbers satisfying $0<r<R$. Thus, by (5), $z \in U_{1}$ and $w \in U_{2}$ are identified if and only if $z=\frac{\varepsilon r}{R} w$. Similar reasoning as in example 1 at once shows

$$
\omega_{\varepsilon}(x, y)=[\mathscr{P}(\ln x / y)-\eta] \frac{d x d y}{x y}, \quad x, y \in S \backslash\left(U_{1} \cup U_{2}\right)
$$

where $\mathscr{P}(z)=\mathscr{P}(z ; 2 \pi i, \ln \varepsilon r / R)$ is the Weierstrassian pe-function with $\eta=\frac{1}{2 \pi i}(\zeta(z+2 \pi i)-\zeta(z))$. Again, it is well-known (see [4, p. 477]) that $\mathscr{P}(z)$ has an expansion given by

$$
\mathscr{Q}(z)-\eta=\frac{e^{z}}{\left(e^{z}-1\right)^{2}}+\sum_{n=1}^{\infty} \frac{h^{n} e^{-z}}{\left(1-h^{n} e^{-z}\right)^{2}}+\sum_{n=1}^{\infty} \frac{h^{n} e^{z}}{\left(1-h^{n} e^{z}\right)^{2}}
$$

with $h=\varepsilon r / R$. Thus (81) and (82) give

$$
\omega_{\varepsilon}(x, y)=\frac{1}{(x-y)^{2}}+\sum_{n=1}^{\infty} \varepsilon^{n} \sum_{\substack{d \backslash n \\ d>0}} d(r / R)^{n}\left(\frac{y^{d-1}}{x^{d+1}}+\frac{x^{d-1}}{y^{d+1}}\right) .
$$

On the other hand, from (71) and (11) it is seen that

$$
a_{n}^{(1)}(x)=\frac{r^{n}}{x^{n+1}}, \quad a_{n}^{(2)}(x)=-\frac{x^{n-1}}{R^{n}} \quad(n=1,2, \cdots)
$$


and

$$
\alpha_{j k}^{11}=\alpha_{j k}^{22}=0, \quad \alpha_{j k}^{12}=\alpha_{j k}^{21}=-\delta_{j k}(r / R)^{\jmath}, \quad(j, k=1,2, \cdots) .
$$

Hence, by (25)", $\Omega_{n, j k}^{l m}$ 's all vanish except when $l=m$ and $\jmath=k$. In the exceptional case, we have

$$
\Omega_{n, 12}^{d d}=\Omega_{n, 21}^{d d}= \begin{cases}-d(r / R)^{n-d} & \text { if } d \mid n(d<n), \\ 0 & \text { otherwise, }\end{cases}
$$

concluding that (83) agrees with our Theorem 6 .

\section{REFERENCES}

[1] Ahlfors, L. V.-Sario, L., Riemann surfaces, Princeton Uuiv. Press., Princeton, 1960.

[2] Curtiss, J., Faber polynomials and the Faber series, Ann. Math. Mon., 78 (1971), 577-596.

[3] FAY, J.D., Theta functions on Riemann surfaces, Springer Lecture Notes of Math., Vol. 352, 1973.

[4] HANCOCK, H., Lectures on the theory of elliptic functions, John Wiley and Sons, New York, 1910.

[5] Jenkins, J.A., Some area theorems and a special coefficient theorem, Illinois J. Math., 8 (1964), 80-99.

[6] Lebowitz, A., On the degeneration of Riemann surfaces., Advances in the theory of Riemann surfaces, Ann. of Math. Studies., 66 (1971), 265-286.

[7] Milin, I. M., The area method in the theory of univalent functions, Soviet Math., 5 (1964), 78-81.

[8] PATT, C., Variations of Teichmüller and Torelli surfaces, J. d'Analyse Math., 11 (1963), 221-247.

[9] Pommerenke, Ch., Über die Faberschen Polynome schlichter Funktionen, Math. Z., 85 (1964), 197-208.

[10] Schiffer, M.-Spencer, D. C., Functionals of finite Riemann surfaces, Princeton Univ. Press, Princeton, 1954.

[11] WEYL, H., The concept of a Riemann surface, $3^{\text {rd }}$ edition, Addison-Wesley, 1955.

ToKyo Institute of Technology. 